University of Wollongong

Research Online

Faculty of Engineering and Information

Faculty of Engineering and Information

Sciences - Papers: Part A

Sciences

$1-1-2014$

\title{
A review of microfabrication techniques and dielectrophoretic microdevices for particle manipulation and separation
}

\author{
Ming Li \\ University of Wollongong, ml433@uowmail.edu.au \\ Weihua Li \\ University of Wollongong, weihuali@uow.edu.au \\ Jun Zhang \\ University of Wollongong, jz218@uowmail.edu.au \\ Gursel Alici \\ University of Wollongong, gursel@uow.edu.au \\ Weijia Wen \\ Hong Kong University of Science and Technology
}

Follow this and additional works at: https://ro.uow.edu.au/eispapers

Part of the Engineering Commons, and the Science and Technology Studies Commons

Research Online is the open access institutional repository for the University of Wollongong. For further information contact the UOW Library: research-pubs@uow.edu.au 


\title{
A review of microfabrication techniques and dielectrophoretic microdevices for particle manipulation and separation
}

\begin{abstract}
The development of lab-On-A-Chip (LOC) devices over the past decade has attracted growing interest. LOC devices aim to achieve the miniaturization, integration, automation and parallelization of biological and chemical assays. One of the applications, the ability to effectively and accurately manipulate and separate micro- and nano-scale particles in an aqueous solution, is particularly appealing in biological, chemical and medical fields. Among the technologies that have been developed and implemented in microfluidic microsystems for particle manipulation and separation (such as mechanical, inertial, hydrodynamic, acoustic, optical, magnetic and electrical methodologies), dielectrophoresis (DEP) may prove to be the most popular because of its label-free nature, ability to manipulate neutral bioparticles, analyse with high selectivity and sensitivity, compatibility with LOC devices, and easy and direct interface with electronics. The required spatial electric non-uniformities for the DEP effect can be generated by patterning microelectrode arrays within microchannels, or placing insulating obstacles within a microchannel and curving the microchannels. A wide variety of electrode- and insulator-based DEP microdevices have been developed, fabricated, and successfully employed to manipulate and separate bioparticles (i.e. DNA, proteins, bacteria, viruses, mammalian and yeast cells). This review provides an overview of the state-of-the-art of microfabrication techniques and of the structures of dielectrophoretic microdevices aimed towards different applications. The techniques used for particle manipulation and separation based on microfluidics are provided in this paper. In addition, we also present the theoretical background of DEP.
\end{abstract}

\section{Keywords}

manipulation, particle, separation, review, microdevices, microfabrication, techniques, dielectrophoretic

\section{Disciplines}

Engineering | Science and Technology Studies

\section{Publication Details}

Li, M., Li, W. H., Zhang, J., Alici, G. \& Wen, W. (2014). A review of microfabrication techniques and dielectrophoretic microdevices for particle manipulation and separation. Journal of Physics D: Applied Physics, 47 (6), 1-29. 


\title{
A review on microfabrication techniques and dielectrophoretic microdevices for particle manipulation and separation
}

\author{
M. $\mathrm{Li}^{1}$, W.H. Li ${ }^{1}$, J. Zhang ${ }^{1}$, G. Alici ${ }^{1}$, and W. Wen ${ }^{2}$ \\ ${ }^{1}$ School of Mechanical, Materials and Mechatronic Engineering, University of Wollongong, \\ Wollongong, NSW 2522, Australia \\ ${ }^{2}$ Department of Physics, The Hong Kong University of Science and Technology, Clear Water Bay, \\ Kowloon, Hong Kong \\ *Corresponding author:weihuali@uow.edu.au
}

\begin{abstract}
The development of lab-on-a-chip (LOC) devices over the past decade has attracted more and more interest, and aims to achieve the miniaturization, integration, automation, and parallelization of biological and chemical assays. One of the applications, the ability to effectively and accurately manipulate and separate micro- and nano-scale particles in an aqueous solution is quite appealing in biological, chemical and medical fields. Among the technologies that have been developed and implemented in microfluidic microsystems for particle manipulation and separation, such as mechanical, inertial, hydrodynamic, acoustic, optical, magnetic, and electrical methodologies, dielectrophoresis (DEP) may prove to be the most popular method, because of its label-free nature, ability to manipulate neutral bioparticles, analyse with high selectivity and sensitivity, compatibility with LOC devices, and easy and direct interface with electronics. The required spatial electric nonuniformities for the DEP effect could be generated by patterning microelectrode arrays within microchannels, or placing insulating obstacles within a microchannel and curving the microchannels. A wide variety of electrode- and insulator-based DEP microdevices have been developed, fabricated, and successfully employed to manipulate and separate bioparticles (i.e. DNA, proteins, bacteria, viruses, mammalian and yeast cells). This review provides an overview of the state-of-the-art microfabrication techniques and structures of dielectrophoretic microdevices aimed towards different applications. The techniques used for particle manipulation and separation based on microfluidics are provided, and the theoretical background of DEP is presented.
\end{abstract}




\section{Introduction}

The development of microfluidic platforms is heading towards the miniaturisation, integration, and automation of biological and chemical assays [1,2]. Lab-on-a-chip (LOC) devices are direct representatives of such a trend, which are also known as micro total analysis systems ( $\mu$-TAS) that integrate laboratory functions on a single small scale chip $[3,4]$. Their small volume reduces the time required to synthesize and analyse, as well as less volumes of fluid required and lower reagent costs; the faster response of the microsystem allows for greater control of reaction process; compact devices achieved massive parallelisation and high-throughput analysis; lower fabrication costs contribute to the mass production of disposable chips. The features of compactness, portability, sensitivity, and parallelisation make LOC devices beneficial for various applications, including point-of-care (POC) diagnostics, drug delivery, molecule and material synthesis, chemical reaction control and detection, cellular process studies and environmental monitoring. One of the existing applications is to manipulate and separate particles (both synthetic and biological), which plays an important role in the field of biology, chemistry and medicine.

To date, a variety of technologies have been developed to manipulate and separate particles in an aqueous solution, including mechanical, inertial, hydrodynamic, acoustic, optical, magnetic, and electrical methodologies [5,6,7]. Among these available methods, dielectrophoresis (DEP) has attracted most attention due to its great advantages [8,9]. Unlike other techniques, DEP depends on the dielectric properties, which represent the structural, morphological, and chemical characteristics of bio-particles, allowing highly selective and sensitive analysis. DEP manipulation is straightforward and fully controllable by varying the electric conductivity of the suspending medium or the frequency and magnitude of the electric field applied. DEP is also easily and directly interfaced to conventional electronics, and can be used in the fabrication of LOC devices. In addition, DEP enables contact-free manipulation of particles (both charged and neutral) with lower sample consumption and fast speed. DEP, first adopted by Pohl [10], is a phenomenon that occurs due to a translational force exerted on a dielectric particle in a non-uniform electric field. A dielectric particle and the suspending medium become electrically polarised in an electric field, which separates the 
electric charge at the interface between solid and liquid. Depending on the relative polarizability of the particle and suspending medium, a net dielectrophoretic force will attract the particle towards the region of higher electric field gradient (positive DEP) or push the particle towards the region of lower electric field gradient (negative DEP) [11]. DEP has proven to be a versatile mechanism for manipulating various micro/nano scale bio-particles (i.e. cells, viruses, bacteria, DNA and proteins) in microfluidic systems, which have been reviewed in the relevant literature $[11,12,13,14,15,16,17,18]$. However, these review articles are mainly focused on theory of DEP and applications of DEP-based microfluidic systems. Although Martinez-Duarte [19] provided a review of microfabrication technologies for dielectrophoretic applications, an in-depth overview of techniques for fabricating integrated dielectrophoretic-microfluidic devices and the correspondingly created DEP devices of different structures and configurations has not yet been conducted.

There are two main methods used to generate the electric field gradient required for the DEP effect: microelectrodes and insulator structures. Traditionally, high-frequency alternating current (AC) electric fields were applied via two-dimensional (2D) or three-dimensional (3D) microelectrode arrays embedded within the microchannels. The 2D planar microelectrodes were normally patterned on the bottom floor of the microchannel by the photolithograph, thin-film deposition and lift-off techniques, which could affect the movement of particles close to the surface of the electrode [11]. Alternatively, 3D microelectrode structures [14] such as extruded vertical electrodes on the bottom, top-bottom patterned, and sidewall patterned microelectrodes have been developed and fabricated using more complicated techniques (i.e. electroplating and pyrolysis), which could generate DEP forces over a larger volume within the microchannel. Alternatively, inhomogeneous electric fields could be induced by insulator structures [20] normally fabricated by soft lithograph technique, in which either insulating obstacles are embedded within the microchannel or the geometry of the insulating microchannel is modified, while direct current (DC) or DC biased lowfrequency $\mathrm{AC}$ electric fields are applied via external electrodes placed in the inlet and outlet reservoirs. The microfabrication techniques currently available have been applied to build electrode- and insulator-based dielectrophoretic microdevices. With the rapid development of LOC or $\mu \mathrm{TAS}$ in recent years, various microfabrication techniques have been developed and applied for dielectrophoretic applications, and dielectrophoretic microdevices with different configurations and structures of microelectrodes and insulators have been successfully used to manipulate and separate particles. 
This paper is organized as follows: we first review the techniques for particle manipulation and separation in an aqueous solution. Subsequently, the concepts and theoretical background of DEP are presented. Then, the microfabrication techniques are reviewed according to their applications for electrode- and insulator-based DEP microdevices followed by different DEP microdevices classified depending on the structures of electrodes (i.e. 2D and 3D), and insulators (i.e. obstacles and curved channels).

\section{Particle manipulation technologies}

Manipulating and separating particles is a critical activity in a variety of biological, medical, and biochemical applications. However, traditional techniques are labour intensive, and require multiple additional tags or labels to identify target particles. Fluorescence-activated cell sorting (FACS) $[21,22,23]$ and magnetic-activated cell sorting (MACS) $[24,25,26]$ utilize fluorophore-conjugated antibodies and magnetic beads conjugated with antibodies to label cell of interests, respectively. Although both methods offer high-throughput screening with rich data outputs, they are costly in terms of system setup, operation and reagent, and are prone to damage on cell fate and function due to the use of fluorophores and antibodies. Moreover, centrifugation is a common technique to separate particles in bulk based on their differences in size and density [27], but this manual, macroscale, multistep technique needs laboratories dedicated to these functions and may introduce contamination during the process [28]. Recently, there has been an interest in label-free techniques that take advantage of intrinsic properties of particle populations, such as size, shape, electrical polarizability, and density to accomplish particle/cell manipulation and separation in fluid, which involve mechanical, inertial, hydrodynamic, acoustic, optic, magnetic, and electrical methodologies.

(1) Mechanical manipulation of bioparticles (i.e. blood cells [29,30], plasma [31,32], and circulating tumor cells [33]) mechanically based on size has been achieved by fabricating constriction structures (i.e. weir [34], pillar [35], and through-holes [36]) within the microchannels. Although these filter-based microfluidic devices had several advantages such as high labelling efficiency and reproducibility, there are many challenges including the heterogeneity of cell sizes within a population, clogging, fouling, and limited selectivity for biological assays. 
(2) Inertial lift forces acting particles in high-velocity flows have been utilized to manipulate polystyrene particles, cells and bacteria within various-shaped microchannels, such as curve [37], asymmetrically curve [38,39], spiral [40] microchannles, a microchannel consisting of a focusing, expansion and collection region [41], and a multi-orifice microchannel [42]. The inertial separation offers high volumetric flow rates, but it requires dilution either prior to injection or within the device to eliminate particle-particle interactions, and a long channel for effective focusing performance due to the increased viscosity.

(3) The hydrodynamic technique presumes that particles will follow the fluid streamlines at a low Reynolds number, which has been applied within microchannels having multiple side branch channels [43], a pinched segment [44], or micro-obstacle arrays [45], and the separation of different-sized polystyrene particles [45], leukocytes from erythrocytes by size [46], blood plasma from the blood cells [47], and healthy lymphocytes from malignant lymphocytes [48] have been accomplished.

(4) The acoustic technique based on the concept that particles suspended in fluid experience an acoustic radiation force when they are exposed to an ultrasound. There are several reports regarding the application of an ultrasonic standing wave field combined with laminar flow to separate different-sized particles [49,50], blood cells [51], and yeast cells [52].

(5) Optical tweezers could tether biological molecules to particles, and then capture the particles at the focal point of an electric field gradient, which has been widely used as a tool for the manipulation and trapping of particles [53,54,55], however, its application in microfluidics is still limited due to the complicated setup, complex operation, and expensive instrumentation.

(6) Magnetic manipulation is a passive and label-based method that achieved by selectively bonding cells with magnetic beads, and then separating them by applying a magnetic field, in addition, the intrinsic magnetophoretic properties of particles or cells (or magnetophoresis) have been employed for separation and purification. The separation of red and white blood cells from whole blood [56,57,58], and living cells loaded with magnetic nanoparticles [59] have been achieved. 
(7) Two main kinds of electrical forces, i.e. Coulomb force and dielectrophoretic force, have been used to manipulate particles. A Coulomb force is generated by interaction between the net charge on the particle and the applied electric field, and the induced movement of the charged particle is known as electrophoresis (EP). Several studies have demonstrated the biological applications of EP, and were reviewed by Korohoda and Wilk [60]. However, for biological particles that are electrically neutral, EP may not applicable due to the absence of net charge, whereas a DEP force that arises from the interaction between the particle's dipole and a nonuniform electric field, can be used to manipulate neutral particles. With the rapid development of lab-on-a-chip (LOC) devices recently, DEP has been widely used for particle sorting, focusing, patterning and positioning due to its many advantages.

\section{Dielectrophoresis (DEP)}

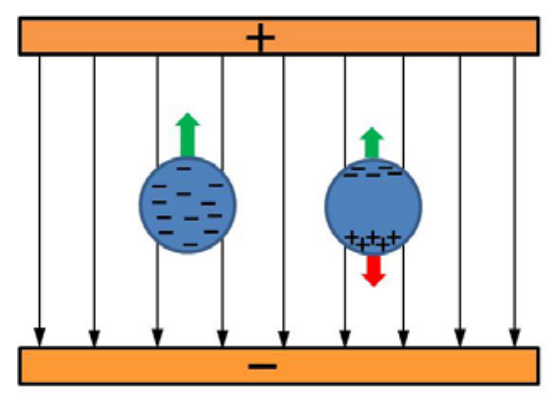

(a)

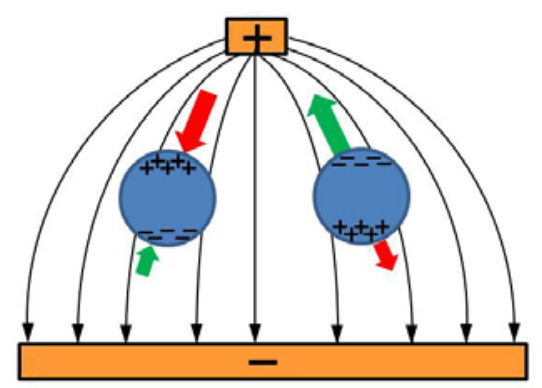

(b)
Microelectrodes
$\rightarrow$ Electric field lines
Particles
Positive forces
\pm Charges
$\Rightarrow$ Negative forces

Figure 1 Principle of dielectrophoresis. (a) The behavior of particles (charged and neutral) in a uniform electric field. (b) The behavior of neutral particles in a non-uniform electric field, which experience net forces.

When an electrically charged and an electrically neutral particle are placed in a uniform electric field (Fig. 1a), they behave differently due to different ion/charge dispersion processes. For the former, it is surrounded by a diffuse layer which contains an excessive number of mobile ions of an opposite charge than that of the like charge, which forms an well-known electric double layer (EDL). The charged particle moves towards the electrodes of opposite electrical polarity due to the Coulomb force (or electrophoretic force). For the neutral particle, electric charges are redistributed within the dielectric particle and in the liquid side of the solid-liquid interface, building up a dipole moment. However, the Coulomb forces on each half of the dipole moment are equal in magnitude and opposite in direction, 
resulting in a zero net force on the particle. As shown in Fig. 1b, when the neutral particle is placed in a nonuniform electric field, the Coulomb forces on either sides of the dipole moment can be different, resulting in a net force exerting on the particle, which is known as dielectrophoretic force. The direction of the force depends on the relative polarizabilities of the particle and the suspending medium, which induces the particle to move towards or against the region of electric field maxima, such a motion is called dielectrophoresis [10]. The dipole moment of a spherical particle is given by $[61,62]$

$$
\mathbf{p}=4 \pi \varepsilon_{m} r^{3} K(\omega) \mathbf{E}
$$

where the bold letters refer to a vector quantity, $\varepsilon_{m}$ is the absolute permittivity of the suspending medium, and $r$ represents the particle radius, Re indicates the real part. $K(\omega)$ is the Clausius-Mossotti (CM) factor, which depends on the complex permittivities of the particle and the suspending medium, as well as the frequency of the external electric field:

$$
K(\omega)=\frac{\varepsilon_{p}^{*}-\varepsilon_{m}^{*}}{\varepsilon_{p}^{*}+2 \varepsilon_{m}^{*}}
$$

where $\varepsilon^{*}=\varepsilon-i \sigma / \omega(i=\sqrt{-1})$ is the complex permittivities, $\sigma$ is the electrical conductivity; $\omega$ is the frequency of the electric field. The subscripts $p$ and $m$ denote the particle and suspending medium, respectively. The complex permittivities are presented, because both the particles and suspending medium exhibit dielectric and conductive properties in the presence of an electric field.

In terms of the dipole moment, $\mathbf{p}$, the time-average dielectrophoretic force acting on a spherical particle in a nonuniform electric field can be written as $[61,62]$

$$
\mathbf{F}_{D E P}=\mathbf{p} \nabla \mathbf{E}=2 \pi \varepsilon_{m} r^{3} \mathbf{R e}[K(\omega)] \nabla \mathbf{E}^{2}
$$

Eqn. 3 implies that the DEP force depends on a variety of parameters such as particle size, the permittivities and conductivities of the particle and suspending medium, and the spatial nonuniformity of the electric field. Moreover, the CM factor plays an important role in DEP force, which represents the dielectric properties of the DEP environment from the contribution of the particle and suspending medium. It also shows that the DEP effect 
depends on the frequency of external electric fields, which is considered to be an excellent feature of this phenomenon. If the particle is more polarisable than the suspending medium $(\boldsymbol{R e}[K(\omega)]>0)$, the particle is pushed towards the region of strong electric field, and such a motion is termed positive DEP (p-DEP). In contrast, if the suspending medium is more polarisable than the particle $(\operatorname{Re}[K(\omega)]<0)$, the motion of the particle is away from the region of strong electric field, which is called negative DEP (n-DEP) [63].

The frequency dependence of the DEP force is shown by investigating the CM factor of a homogeneous spherical particle, i.e. Eqn. 2 . The factor can vary from -0.5 (i.e. $\varepsilon_{p}^{*}<<\varepsilon_{m}^{*}$ ) to +1 (i.e. $\varepsilon_{p}^{*}>>\varepsilon_{m}^{*}$ ), which means a positive DEP force can be twice as strong as a negative DEP force. Moreover, at low and high frequency limits, the CM factor is reduced to the following forms, respectively

$$
\left\{\begin{array}{c}
K(\omega)=\frac{\sigma_{p}-\sigma_{m}}{\sigma_{p}+2 \sigma_{m}}, \text { for } \omega \rightarrow 0 \\
K(\omega)=\frac{\varepsilon_{p}-\varepsilon_{m}}{\varepsilon_{p}+2 \varepsilon_{m}}, \text { for } \omega \rightarrow \infty
\end{array}\right.
$$

Therefore, at low frequencies, the DEP force depends on the conductive properties of the particle and the suspending medium, while the permittivity values govern the DEP effect at high frequencies. At intermediate frequencies, both the dielectric and conductive properties of the particle and suspending medium determine the magnitude and polarity of the DEP force. When the electric conductivity of particle is fixed while the frequency of applied electric field is varied, the particle may have different DEP response [14]. At a range of frequencies, the particle experiences a positive DEP, while exhibits a negative DEP response at another range of frequencies. The point where the response switches from p-DEP to n-DEP or from n-DEP to p-DEP is called crossover frequency. At this frequency, the complex permittivity of the particle is equal to that of suspending medium, and the DEP force will be zero $(\boldsymbol{R e}[K(\omega)]=0)$. The shift of the crossover frequency could be achieved by changing the permittivity and conductivity of the particle and suspending medium.

In Eqn. 3, the DEP force induced in DC fields and stationary AC fields have been considered, but if the phase of the AC field has a spatial variation, the time-averaged DEP force arising 
from the interaction of the induced dipole moment and the phase of the various field components should be taken into account. Hence, the time-averaged DEP force can be rewritten as

$$
\mathbf{F}_{D E P}=2 \pi \varepsilon_{m} r^{3} \mathbf{R e}[K(\omega)] \nabla \mathbf{E}^{2}+4 \pi \varepsilon_{m} r^{3} \mathbf{I m}[K(\omega)] \sum \mathbf{E}^{2} \nabla \varphi
$$

where Im indicates imaginary part, $\varphi$ is the phase component of the AC field. The second term relates to the imaginary part of the $\mathrm{CM}$ factor and the spatial nonuniformity of the field phase, which is called a 'travelling wave DEP (twDEP)'. This force directs the particle against or along the direction of travel of field according to the polarity of $\operatorname{Im}[K(\omega)][64]$. As shown in Fig. 2, a series of planer electrodes are exited with potentials having a $90^{\circ}$ phase variation between the adjacent ones, which induces co-/anti-field twDEP response directing the particle towards/ off the smaller phase regions.

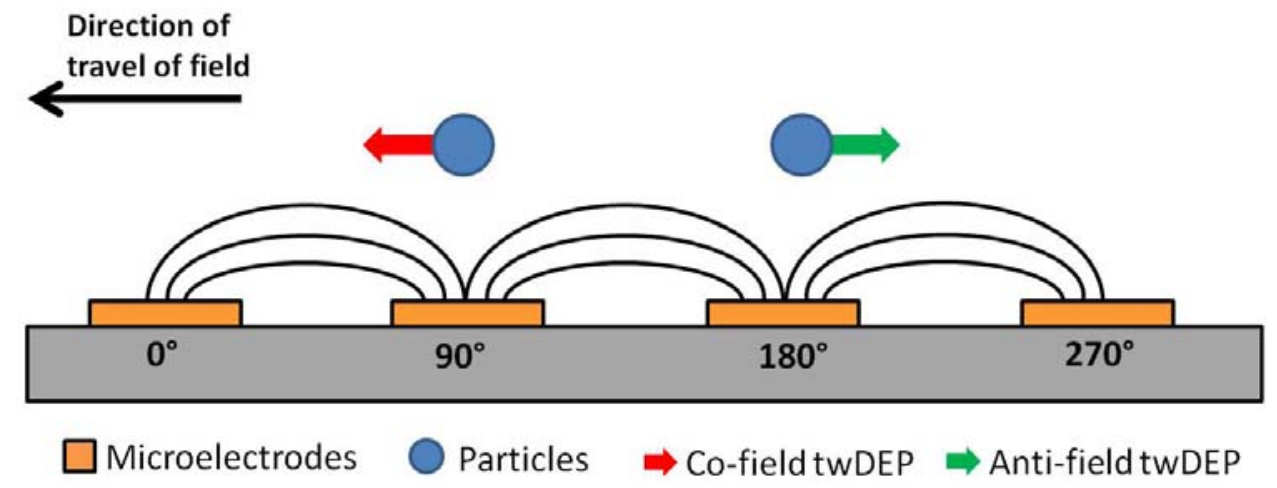

Figure 2 Schematic illustration of travelling wave dielectrophoresis (twDEP) of a spherical particle.

When a DC field (the frequency is zero) is applied, the DEP force expression (Eqn. 5) remaines the same, but the $\mathrm{CM}$ factor only depends on the electrical conductivities of the particle and the medium, and is simplified into Eqn. 4a $[15,65]$. The particle suspended in liquid also experiences an electrokinetic (EK) effect which is a combination of fluid electroosmosis (EO) and particle electrophoresis (EP) [61]. The resulting motion is determined by the electrokinetic velocity of the particle, which is linearly proportional to the local electric field and leads to the particle being transported along the electric lines. Therefore, the motion of the particle in a DC electric field is governed by both the linear electrokinetic effect and the nonlinear dielectrophoretic effect. 
Unlike electrophoresis, which relies on the net electrical charge of particles, dieletrophoresis depends on their dielectric properties which enables it to manipulate neutral bioparticles [10]. As the dielectric properties represent many structural, morphological and chemical characteristics of the particles, DEP offers a more selective and sensitive analysis of biological samples [63]. DEP manipulation is also straightforward and fully controllable by varying the electrical conductivity of the suspending medium or the frequency and magnitude of the applied electric field, and a label-free manipulation of particles with lower sample consumption and fast speed can be achieved [8]. In addition, DEP can be easily employed in the fabrication of LOC devices for various applications [9].

\section{Microfabrication techniques for dielectrophoretic-microfluidic devices}

Researchers have generated spatial nonuniformities by using microelectrode arrays embedded within microchannels, and in some cases insulator structures to manipulate and separate particles. Some basic microfabrication techniques, such as photolithography, thin-film deposition, etching, electroplating, soft-lithography, and substrate bonding, etc., have been used to build dielectrophoretic microdevices. The main microfabrication techniques are summarized and presented below based on their applications in DEP-based devices having different electrode or insulator structures.

\subsection{Two-dimensional (2D) planar electrodes}


(a)

(i) Spin-coating

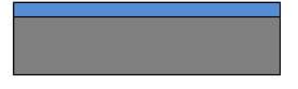

whentwhtwhth

(ii) Expose

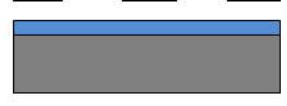

(iii) Develop

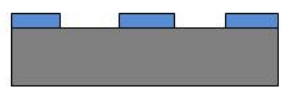

(iv) Deposition

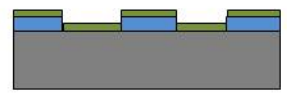

(v) Lift-off

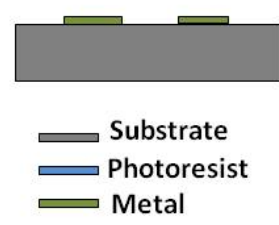

(b)

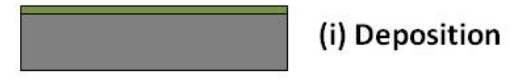

(ii) Spin-coating

\section{whwtwwwtww}

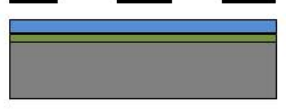

(iii) Expose

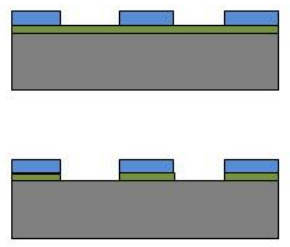

(iv) Develop

(v) Etching

(vi) Strip PR

Figure 3 Fabrication process flow for planar electrodes.

Traditionally, spatial nonuniformity is generated by applying AC electric field via microelectrode arrays patterned within microchannels. The fabrication of 2D planar metal electrodes always required processes that include photolithography, thin-film deposition, and lift-off and/or etching. As shown in Fig. 3a, the mask with the defined microelectrode patterns is firstly designed using AutoCAD or L-edit, after which a positive photoresist is deposited onto a substrate (i.e. glass and silicon) and spin-coated to a final thickness that depends on the spin speed. After the coating, soft-bake is conducted to remove the solvent and improve adhesion to the substrate. The substrate is then aligned to the mask and exposed to a UV source, which is followed by development to obtain the microelectrode lines. As the photoresist is positive, UV-exposed regions will be removed from the substrate, leaving inverse patterns uncovered with photoresist, where the target metal material is to be located. This so-called photolithograph technique is where geometric microelectrode patterns are transferred from a mask to a light-sensitive photoresist. The next process is thin-film deposition, metal of a variety of materials (i.e. gold, platinum and chromium) is deposited on the whole substrate using the evaporation or sputtering technique. In the evaporation technique, the substrate is placed in a vacuum chamber where a piece of metal material is heated, evaporated, and deposited on the top of the substrate. In sputtering, due to the 
bombardment of energetic inert ions, atoms or clusters are ejected from the target material, and then directed towards the substrate. After the metal has been deposited, the substrate is submerged in a solvent (i.e. acetone) to wash off the sacrificial photoresist layer and the target material on its surface. After lift-off, only the target material remains in the regions having a direct contact with the substrate, creating a defined array of microelectrodes. As an alternative (see Fig. 3b), the target metal material is first evaporated or sputtered on the top of the whole substrate, and after the thin-film deposition, a photoresist layer is patterned using the standard photolithography technique mentioned above, which is followed by a wet/dry etching step to selectively remove materials from the substrate. Wet etching always utilizes potassium hydroxide $(\mathrm{KOH})$, ethylene diamine pyrochatechol (EDP), and tetramethyl ammonium hydroxide (TMAH), and shows high material selectivity. In contrast, dry etching technique, such as plasma etching, reactive ion etching, and ion milling, is advantageous in terms of a smaller undercut and higher anisotropicity, which contribute to smaller pattern lines and structures with a high aspect ratio, respectively. Besides, a metal layer could be directly patterned using laser ablation or deposition via a stencil or shadow mask, in which case the photolithograph and etching process are not required.

\subsection{Three-dimensional (3D) electrodes}

Although 2D planar electrodes that commonly patterned on the bottom of the microchannel were developed for various dielectrophoretic applications, using this structure for some practical applications can be problematic. This is because electric field gradient decrease rapidly as the distance from the electrodes increase, so that only the motion of particles close to the electrode surface can be directed, which greatly reduces the efficiency of the microsystems. Moreover, adhesion of the particles on the surface of the electrode or the channel wall is always an issue. These limitations can be avoided by fabricating 3D electrodes. Based on the configuration and structures of in-channel electrodes, 3D electrodes can be classified to top-bottom patterned, extruded from channel bottom, sidewall patterned ones, in addition, the electrodes can be classified based on materials, such as metal, carbon, silicon and polymer composite, which are summarized and discussed as follows.

\subsubsection{Top-bottom patterned electrodes}




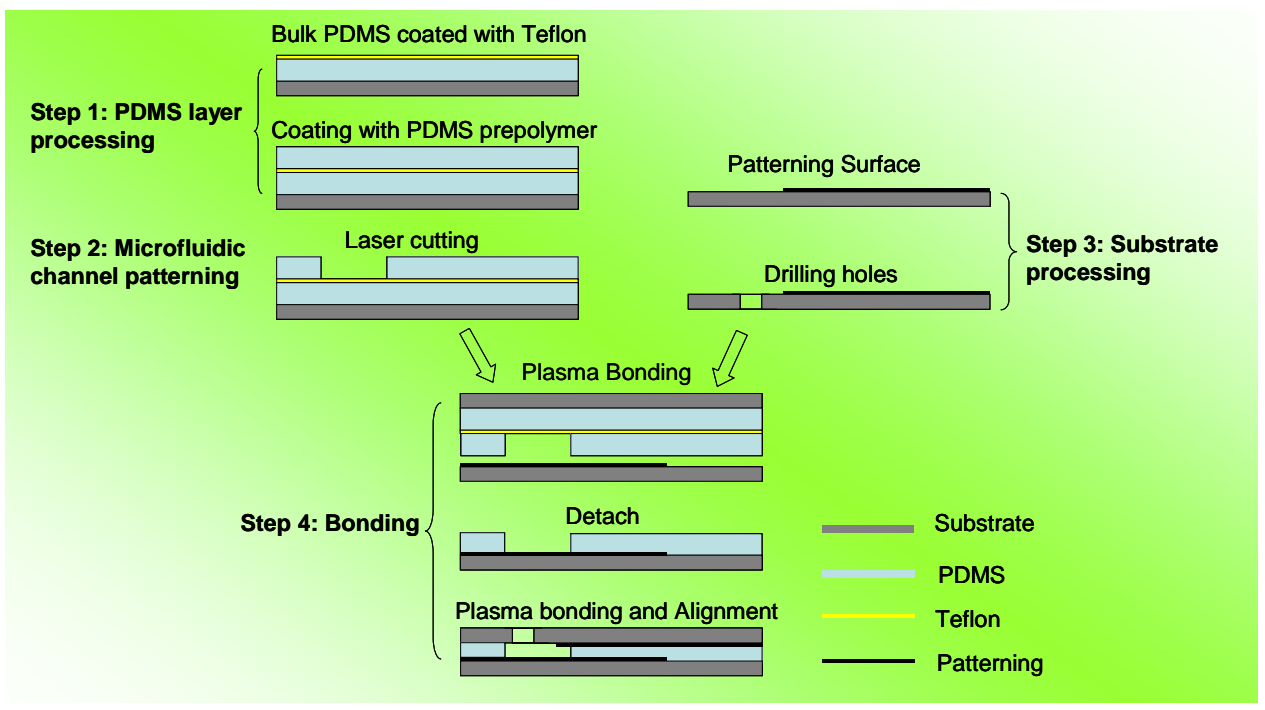

Figure 4 Fabrication process flow for a microfluidic device with top-bottom patterned microelectrodes: (1) PDMS layer process, (2) microfluidic channel patterning, (3) substrate processing, and (4) bonding [66]. Reprinted with permission from M. Li et al., Microfluid. Nanofluid. 12, 751 (2012). Copyright 2011 Springer.

Traditionally, the fabrication process of DEP-based microdevices with three-dimensional (3D) microelectrode arrays patterned on top and bottom surface of the substrate always involves: patterning of planar electrode arrays on both substrates, manufacturing microchannel structure of SU-8 photoresist, and application of a UV curable adhesive $[67,68,69]$ or an epoxy-based adhesive for bonding [70,71], which required standard photolithograph technique to pattern microfluidic channels and exact control of the adhesive thickness to avoid contamination of the microchannel. Alternatively, Li et al [66] proposed using a laser-patterned PDMS layer containing microchannel patterns as an adhesive layer, which was sandwiched between two electrode-patterned substrates (see Fig. 4). Briefly, the fabrication starts with the preparation of two PDMS layers on a glass substrate: one thick layer serves as a transferring layer after being treated with Teflon on its surface, while the other thin layer is a working component of the device, which is also used as an adhesive for bonding with substrates. The thin transferred PDMS layer is then defined with open microchannels using laser ablation method, after which unwanted part of the thin PDMS layer was peeled off and PDMS was cleaned using isopropanol to eliminate ashes and debris created laser. Two substrates were patterned with planar microelectrode arrays and drilled via-holes, and finally bonded with the PDMS layer containing microchannel patterns after oxygen-plasma treatment. 


\subsubsection{Extruded electrodes}

\section{Metal}

The electroplating technique has been used to build extruded metal electrodes inside the channel, where metal ions in a solution are moved by an electric field to coat a conductive metal layer: the part to be plated and the metal needed are the cathode and anode of the circuit, respectively, and both of which are immersed in an electrolyte solution and connected to an external supply of direct current. When a direct current is applied, the metal atoms comprising the anode will be oxidized and dissolved in the solution, which then move toward the cathode and finally plate out onto the cathode. The details of fabricating extruded gold electrodes from the bottom substrates can be found in the work of Voldman et al [72]. Generally, the overall process starts with deposition of two metal layers onto a glass substrate, which is followed by etching the Au layer to define the electrical interconnects. After SU-8 mold patterning and gold posts electroplating, the SU-8 layer and Ti layer were removed. Then, a second SU-8 layer for a flow channel was patterned using the photolithograph technique, holes for fluid access were drilled, and the whole microdevice was enclosed by glue-bonding with a coverslip.

\section{Carbon}

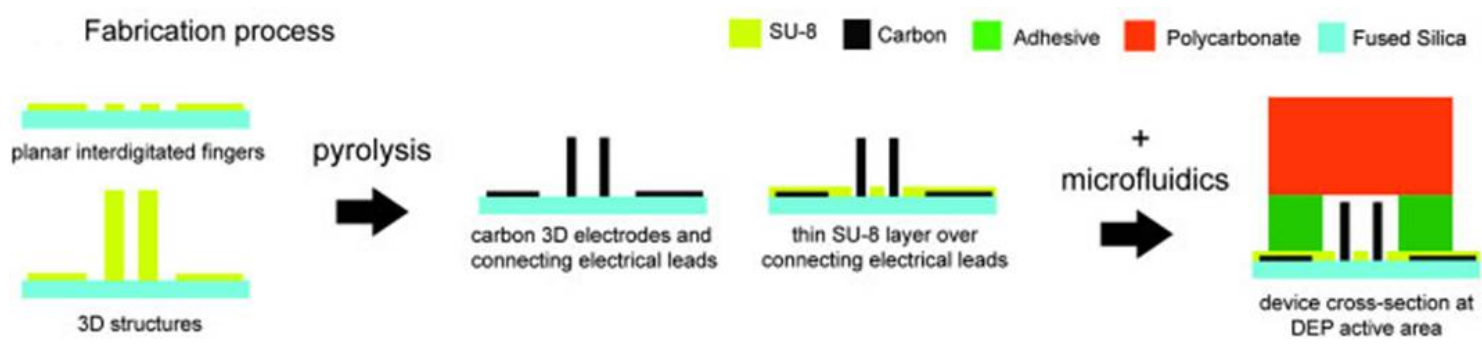

Figure 5 Fabrication process flow for a DEP-based microdevice with carbon electrodes [73]. Reprinted with permission from R. Martinez-Duarte et al., Electrophoresis 32, 2385 (2011). Copyright 2011 WILEY-VCH Verlag GmbH \& Co. KGaA, Weinheim.

The technique utilized to fabricate extruded carbon microelectrodes is called pyrolysis, in which a previously patterned organic precursor is carbonized. Pyrolysis refers to the thermochemical decomposition of organic materials at high temperatures in the absence of oxygen. During this technique, organic materials are transformed into gases or liquid, leaving a solid residue known as glassy carbon, which contains carbon and ash. Martinez-Duarte et al [73] provided the details of fabricating carbon electrodes for dielectrophoresis applications. As shown in Fig. 5, a SU-8 precursor is patterned by photolithography in two steps: a planar 
layer is firstly fabricated for connection leads to the base of the volumetric electrodes, and a pillar array is then fabricated for 3D carbon electrodes. The SU-8 patterns are then carbonized in a furnace under constant nitrogen flow in two stages: removal of residual oxygen in the chamber at $200{ }^{\circ} \mathrm{C}$, further ramp to $900{ }^{\circ} \mathrm{C}$, and dwell at this temperature for 1 hour. The microfluidic network is fabricated separately using plotted double-sided adhesive and polycarbonate (PC) with holes drilled beforehand.

\section{Silicon}

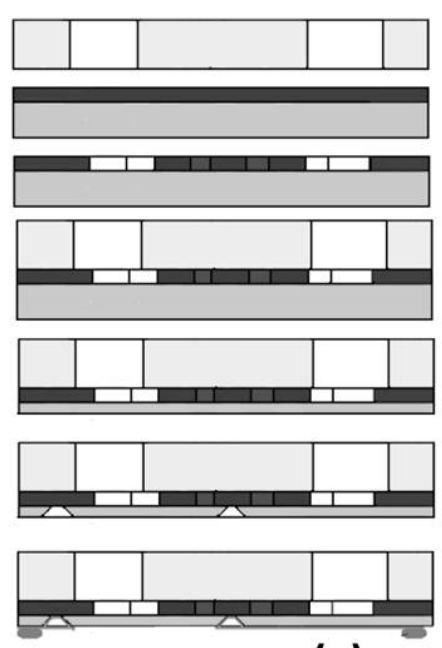

(a)
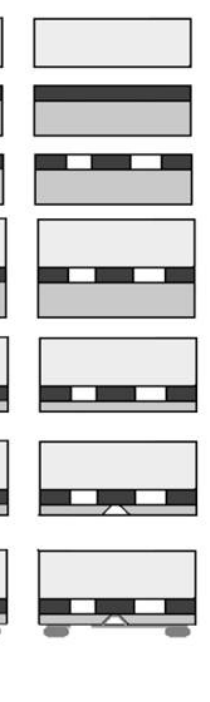

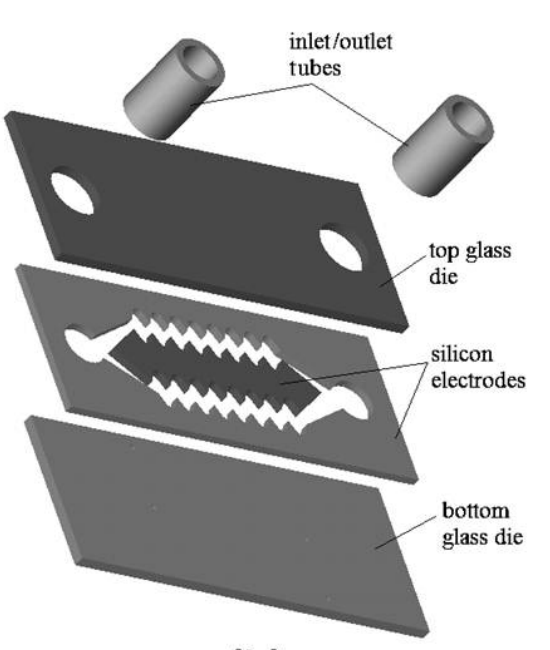

(b)

Figure 6 (a) Fabrication process flow for a DEP-based device with silicon electrodes, and (b) schematic diagram of chip configuration [74]. Reprinted with permission from C. Iliescu et al., J. Micromech. Microeng. 15, 494 (2005). Copyright 2005 IOP Publishing Ltd.

Another type of extruded microelectrodes is silicon electrodes, which are formed by a heavily doped silicon wafer and act as the channel walls in the same time. The details of fabricating a dielectric chip with 3D silicon chip can refer to the work by Iliescu et al [74], where the fabrication process involves anodic bonding, deep reactive ion etching (DRIE), wet etching and thin-film deposition. The fabrication process of this dielectrophoretic device with 3D silicon electrodes is shown in Fig. 6a. A Pyrex glass wafer is first drilled using diamond drill bit to create holes for fluidic access. The silicon-glass anodic bonding of a P-type, heavy doped silicon wafer to a Pyrex glass wafer is performed at $305{ }^{\circ} \mathrm{C}, 1000 \mathrm{~V}$, and $1000 \mathrm{mbar}$, after which the electrodes and microfluidic channel are simultaneously defined within the silicon wafer using the DRIE technique. In this technique, photoresist is first patterned onto the silicon using a photolithograph, which is followed by a stop-etch on the glass substrate 
using SF6/O2 in the etching step and C4F8 in the passivation step. After the photoresist layer has been removed, a second anodic bonding is conducted for the previously prepared glass substrate with drilled holes and the patterned silicon wafer at $450{ }^{\circ} \mathrm{C}, 1500 \mathrm{~V}$, and 2000 mbar. Via-holes are required at the bottom glass substrate for electrical connection, so all other front sides of the process wafer are protected with wax and the bottom glass is thinned to $100 \mu \mathrm{m}$ by dipping it into a $\mathrm{HF}(49 \%) / \mathrm{HCl}(30 \%) 10: 1$ solution. The $\mathrm{Cr} / \mathrm{Au}$ layers are deposited and patterned by wet etching to serve as the mask for creating the via-holes. After the holes have been fabricated by dipping in the $\mathrm{HF} / \mathrm{HCl}$ solution again, the metal mask is removed using classical chromium and gold etchants. Finally, metal leads are fabricated by depositing chromium and gold, patterning the photoresist as a mask, and wet etching to obtain metal patterns. The schematic illustration of the device configuration is shown in Fig. $6 b$.

\subsubsection{Sidewall patterned electrodes}

\section{Metal}

The electroplating technique was also utilized by to implement arrays of 3D vertical electrodes on the sidewalls of the microchannel, and the details of the fabrication process can be found in the work of Wang et al [75]. Briefly, it commences by spin-coating a thin layer of SU-8 and then flood the UV exposure to crosslink it. This thin SU-8 layer could improve the adhesion of the channel layer to the substrate, and also serve as an insulating layer for metal deposition. Thin films of $\mathrm{Ti} / \mathrm{Au}$ are then deposited and patterned to define electrical connection pads, leads, and seed layers for subsequent electroplating. A relatively thick layer of photoresist is photo-patterned with holes to serve as the mold for electroplating electrodes. After that, the electroplating process is carried out by dipping the device into a gold electroplating solution with well controlled stir rates and current densities, where the exposed and developed holes fill with gold. After electroplating, the layer of photoresist is removed and another SU-8 layer is patterned as a microchannel, which is finally sealed with a PDMS sheet and a thin SU-8 layer used as adhesive. 


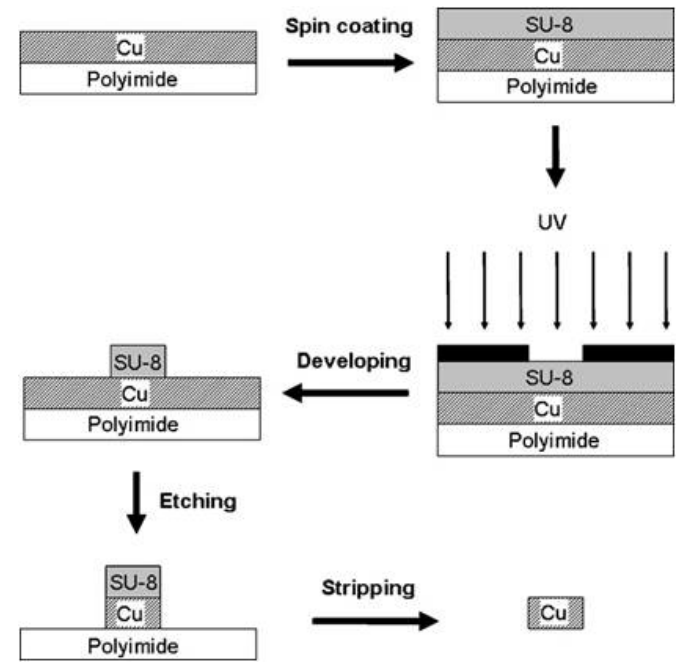

Figure 7 Fabrication process flow for the microscale copper electrodes [76]. Reprinted with permission from Y. Kang et al., Electrochim. Acta 54, 1715 (2009). Copyright 2008 Elsevier.

Another type of sidewall-patterned metal electrodes is micro copper electrodes, the fabrication steps of which are shown in Fig. 7. A layer of SU-8 is spin-coated on the copperclad laminated with a layer of polyimide, which is subsequently patterned using the photolithography technique. The copper sheet is then partly covered with patterned photoresist and dipped into the copper etchant to remove any uncovered copper. The copper electrodes with the desired patterns are released in a bath of $\mathrm{NaOH}(30 \%)$ at $130{ }^{\circ} \mathrm{C}$, while the polyimide substrate and the photoresist are peeled off simultaneously. The microelectrodes are embedded manually into the electrode cavities of a PDMS slab with microchannel patterns, and then the whole component is finally bonded with a glass substrate via plasma treatment. 
(a)

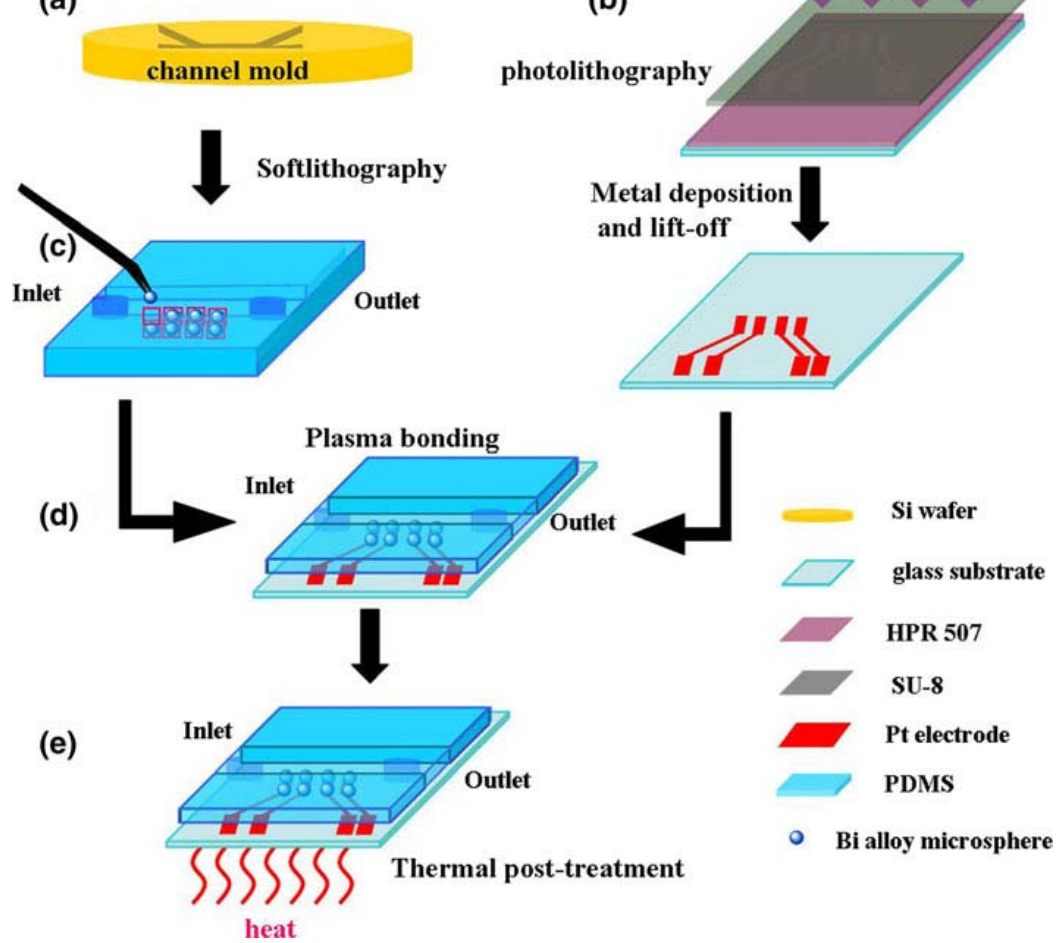

Figure 8 Fabrication process flow for a microfluidic chip with alloy microsphere electrodes embedded at the sidewall of the microchannel: (a) fabrication of SU-8 mold, (b) patterning of planar electrodes, (c) fabrication of microchannel, (d) bonding, and (e) thermal post-treatment [77]. Reprinted with permission from S. Li et al., Microfluid. Nanofluid. 14, 499 (2013). Copyright 2012 Springer.

Alloy microspheres were also used as microelectrodes patterned at sidewalls. As shown in Fig. 8, SU-8 mould for microchannels and deep recesses at sidewalls where microspheres located is patterned using the standard photolithography technique (a), planar microelectrodes for electrical connection are patterned using photolithography, sputtering, and lift-off techniques (b), alloy microspheres formed by a droplet generator are manually positioned in the recesses of the PDMS replica with microchannel patterns (c), bonding is performed between the PDMS layer containing microchannel with metal microspheres and the electrode-patterned bottom substrate by oxygen-plasma treatment (d), thermal post-treatment is carried out in an oven at $60{ }^{\circ} \mathrm{C}$ for $10 \mathrm{~min}$ to improve the electrical contact between the metal microspheres and planar microelectrodes (e).

\section{Liquid electrodes}

In the case of liquid microelectrodes located at the sides of a main flow channel [78], planar metal electrodes are firstly patterned on a substrate by photolithography of resist, thin-film 
deposition, and lift off processes, and then a layer of SU-8 with the desired microchannel patterns and electrode chambers is fabricated onto the top of the microelectrodes using the standard photolithography process. Finally, a PDMS sheet with via-holes and reservoirs is bonded to the pyrex substrate with electrode and microchannel patterns. The fabrication process of liquid electrodes is almost the same as that of 2D planar electrodes at the bottom of the channel, but size of the of the metal electrodes is relatively larger for liquid electrodes in separate side chambers, which leads to longer electrode lifetime, lager interfacial capacities, and lower impedance. Moreover, a novel design — gel electrodes proposed by Luo et al [79] has been applied in DEP microsystems for sorting particles and whole blood. This conductive material can switch between liquid and semisolid, and serve a 3D bridge between planar electrodes and main flow channel, which leads to the improvement of DEP effect and the decrease of electric field gradient dead zones.

\section{Conductive polymer composites}

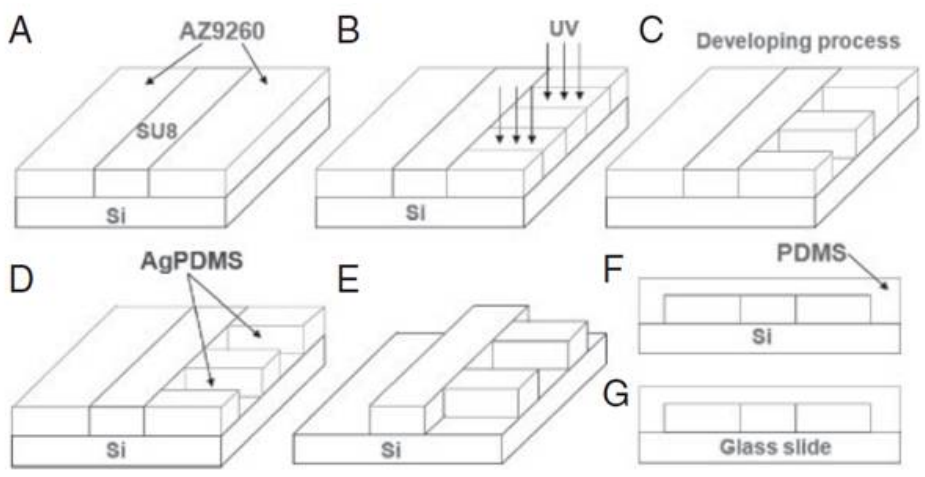

Figure 9 Fabrication process flow for microdevices with 3D conducting PDMS composite electrodes in sidewalls: (A) spin-coating of AZ photoresist, (B) UV exposure, (C) developing, (D) AgPDMS placing, (E) removal of AZ photoresist, (F) curing of PDMS liquid, and (G) bonding [80]. Reprinted with permission from N. Lewpiriyawong et al., Electrophoresis 31, 2622 (2010). Copyright 2010 WILEY-VCH Verlag GmbH \& Co. KGaA, Weinheim.

Conductive polydimethylsiloxane (PDMS) composites have been utilized as sidewall electrodes. Lewpiriyawong et al [80] provided the fabrication process of a dielectrophoretic device with conducting silver-PDMS composites embedded at the sidewall (Fig. 9). A SU-8 layer is first defined for the microfluidic network, and a layer of AZ resists is pattered around the SU-8 patterning using a standard photolithograph technique: spin-coating (A), UV exposure (B) and developing (C). The synthesized AgPDMS composites made from mixing PDMS gel and silver powders at a weight ratio of $85 \%$ is filled the AZ cavities (D), and 
excessive AgPDMS is cleaned by a blade. After the composites have been cured, AZ is removed leaving SU-8 patterning and composite structures on the substrate (E). Pure PDMS gel is poured on the mold, cured, and then peeled off together with AgPDMS composites (F). The PDMS piece is punched to create holes for microfluidic access, treated with oxygenplasma, and bonded to a glass substrate to form enclosed microchannels $(\mathrm{G})$.

\subsection{Insulator structures}

\subsubsection{Glass}

One approach used to fabricate insulator structures is patterning of glass using the wet etching technique. In the isotropic etching of glass, the etchant attacks glass in every direction simultaneously, which results in a semi-circular profile under the mask. Photoresist can serve as the mask for glass etching, but metal masks are always utilized instead to minimize the adverse effect of pin holes on the etching profile. The fabrication process of metal masks includes the metal thin-film deposition and wet/dry etching through a photoresist mask. After etching glass using wet etchants, the mask layer is removed, and thermal bonding is performed in an inert atmosphere such as nitrogen and vacuum, the etched glass hence is sealed to another piece of glass to form a microfluidic network. Insulator-based DEP devices with posts, silica beads and ridges have been fabricated using this process.

\subsubsection{Polymer}

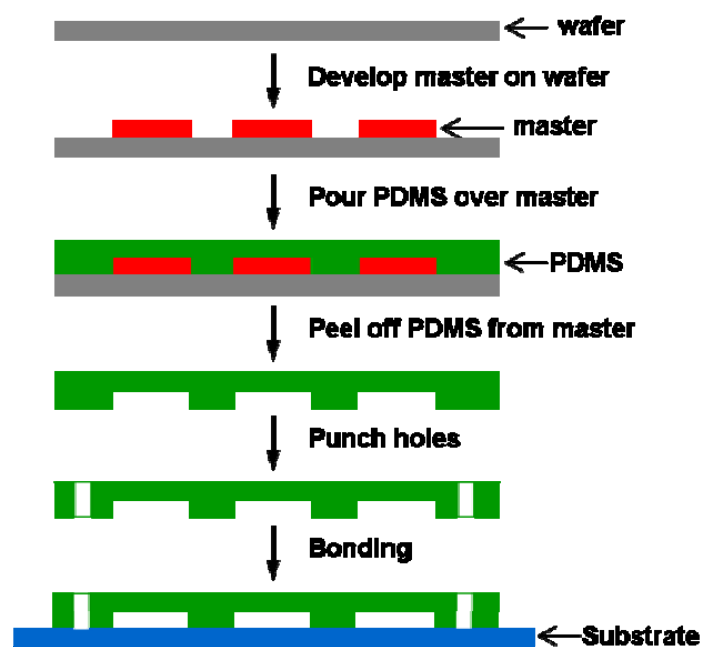

Figure 10 Fabrication process flow for a PDMS-based microfluidic device.

Another type of insulator structures is based on polymer, which has advantages over glass in terms of flexibility and the low-cost fabrication process. The fabrication of patterns and 
structures with polymer is called soft lithography, which is based on injection molding or embossing. In this technique, pattered elastomeric stamps, molds, or conformable photomasks are prepared first, and then a pre-polymer of the elastomer is poured over the master which has relief structures on its surface, cured and peeled off [81]. Among those polymers applied for fabricating microfluidic devices, PDMS is particularly appealing due to its transparency, flexibility, low-cost and compatibility [82,83]. After the patterned PDMS slab has been fabricated, it requires to be bonded with glass, silica, or another piece of PDMS to form an enclosed microfluidic network. The sealing could be achieved by simply oxidizing the PDMS surface without the need for adhesive. Figure 10 illustrates the fabrication process of a microfluidic device based on PDMS, and the fabricating details could be found elsewhere $[84,85]$. Briefly, the fabrication process started with patterning photoresist using the standard photolithography technique to create a positive relief on the surface of a silicon wafer. Once a master has been fabricated, a channel is formed in the PDMS by replica molding, which generates a negative replica of the master in PDMS. Liquid PDMS prepared by mixing Sylgard 184 and curing agent at a weight ratio of 10:1 is poured over the master, degassed, and cured in a vacuum oven. The negative PDMS cast with the microchannel pattern is then peeled off the master, and via-holes for fluidic access or reservoirs for buffer are created. After plasma treatment, the channel side of the PDMS and a substrate (i.e. glass, silicon and PDMS piece) are bonded to form the desired microchannels. DEP devices with obstacles extended from the sidewalls and curved microchannel geometries are fabricated using this technique.

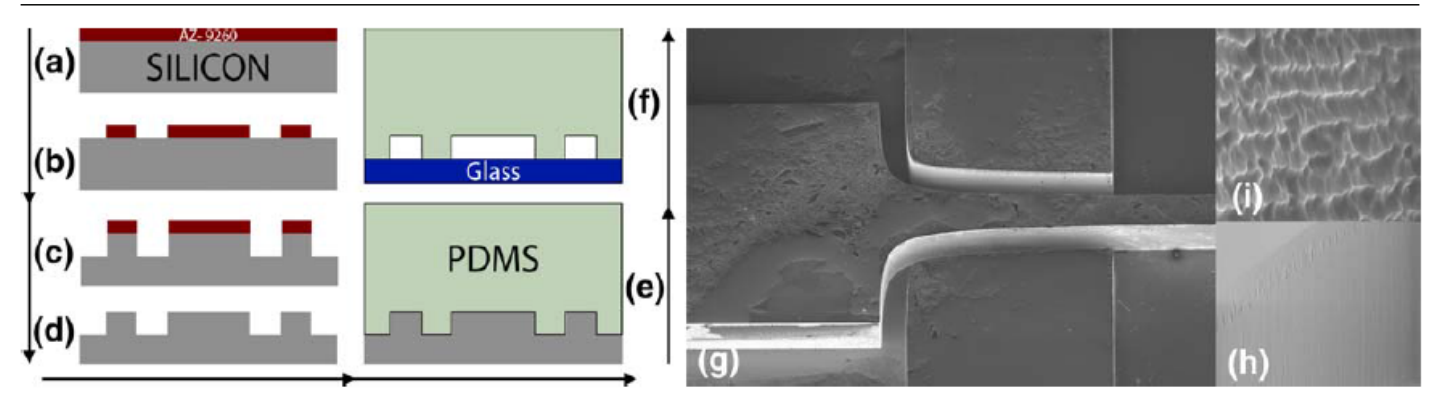

Figure 11 (a-e) Fabrication process flow for microfluidic channels using etched silicon wafers as the master for PDMS casting, (g-h) SEM pictures of the silicon master [86]. Reprinted with permission from H. Shafiee et al., Biomed. Microdevices 11, 997 (2009). Copyright 2009 Springer. 
As an alternative, etched silicon wafers are used as masters for PDMS casting, which requires an additional reactive ion etching (DRIE) step compared to those using patterned resist for the master. The details of fabricating these devices can be found elsewhere [86]. As shown in Fig. 11, AZ photoresist was first spun onto a silicon wafer (a), and patterned using standard photolithography process (b), the silicon master mold with desired depth was then created by DRIE using resists as a mask (c), after removing AZ photoresist (d), liquid PDMS is poured onto the master, degassed, cured, removed (e), and the PDMS replica with punched holes finally bonded to a clean glass slide after plasma treatment (f). Contactless DEP devices $[86,87]$ are always fabricated by the aforementioned process, in which a thin PMDS layer is located between electrode side channels and the main channel to avoid the direct contact between electrodes and sample fluid, therefore, the problems of contamination, bubble formation, mitigates fouling are eliminated.

\subsubsection{Oil}

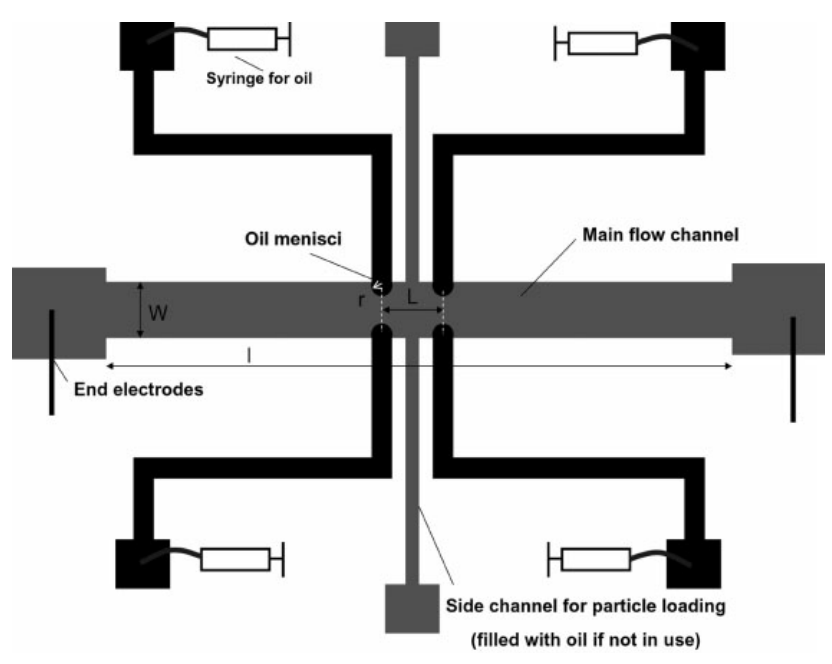

Figure 12 Schematic of the oil-based DEP microfluidic device by applying DC electric field [88]. Reprinted with permission from P. K. Thwar et al., Electrophoresis 28, 4572 (2007). Copyright 2007 WILEY-VCH Verlag GmbH \& Co. KGaA, Weinheim

In addition, insulating oil menisci have been used induce electric field gradient in dielectrophoretic microdevices. The fabrication process of the oil-based DEP devices is similar to that of the insulating polymer-based ones, which involves photolithography to create SU-8 micropatterns as master, soft lithography to replicate microchannel patterns, and plasma bonding to form enclosed channels. The only difference relays on the design of side channels filling with oil, which could be controlled by the connecting syringe pumps. The 
schematic of this design is shown in Fig. 12, where electrode wires are placed in inlet and outlet, and oil menisci are extended into main fluid channel from side channels. This technique provides an alternative method to control the electric field gradient and particle motion within DEP microdevices by adjusting the position, geometry and distance of oil menisci.

Various fabrication techniques used for dielectrophoretic-microfluidic devices have been discussed by classifying electrodes/insulators based on their configurations and compositions. In general, based on the concept of inducing non-uniform electric fields required for DEP effect, DEP devices can be classified to electrode and insulator-based ones, in addition, based on the configuration of electrodes, DEP device can be classified to ones with 2D planar, topbottom patterned, sidewall patterned, 3D extruded electrodes, and a variety of materials including metal, carbon, silicon, conductive polymer composite, glass, insulting polymer and oil have been applied as the elements for electrical field gradient. The fabrication process for each kind of DEP devices is different, and it has its own advantages and disadvantages. For example, the fabrication of 2D planar electrodes on the bottom surface of microchannel is relatively simple, but this design suffers from limited working area of desirable DEP effect. Although 3D electrodes patterned on both top and bottom surfaces of the microchannel can increase the region of effective DEP effect, it suffers difficulties in alignment and bonding steps. Therefore, it is not rigorous to decide which design and fabrication is better or worse, and the choice of optimum design and fabrication process is dependent on the requirement of various applications. The microfabrication techniques of different electrode/ insulator-based dielectrophoretic microdevices are summarized and reported in Table 1.

Table 1 Microfabrication techniques of dielectrophoretic microdevices.

\begin{tabular}{cccc}
\hline Classification & Techniques & Disadvantages & Advantages \\
\hline 2D planar & Photolithography & Limited regions of active & Simple fabrication \\
electrodes & Etching & DEP effect & \\
& Thin-film deposition & & \\
Top-bottom & Photolithography & Alignment and bonding & Large DEP effect region \\
electrodes & Etching & difficulties & High throughput \\
& Thin-film deposition & & High efficiency \\
& Lift-off & & \\
\hline
\end{tabular}




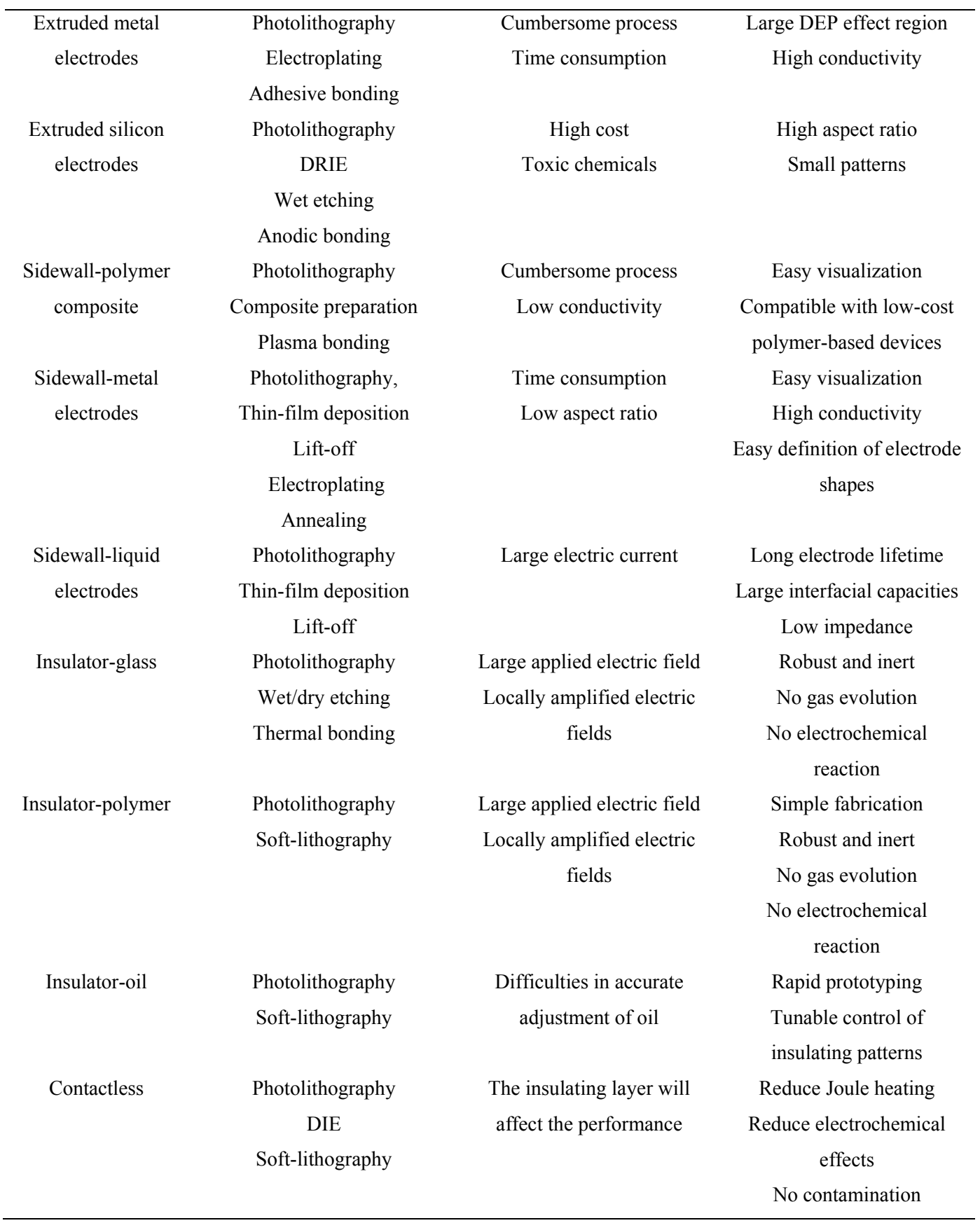

\section{Various DEP microdevices for particle manipulation and separation}

There are two main approaches to create the required inhomogeneous electric field for a DEP effect: (1) microelectrode arrays patterned within the microchannel, which are connected with external high-frequency AC electric fields; and (2) insulating obstacles placed in the microchannel or channel geometry modification, while DC (or AC-biased DC) electric fields are applied to electrodes placing at the inlet and outlet reservoirs. Depending on how the $\mathrm{v}$ microelectrodes or insulators [88]are configured, DEP devices are classified in the following 
main categories: electrode-based (including planar, top-bottom patterned, extruded, and sidewall-patterned, etc.), and insulator-based (including obstacles, curved microchannels, and contactless) dielectrophoretic microdevices.

\subsection{Electrode-based DEP devices}

A variety of two-dimensional (2D) and three-dimensional (3D) microelectrode structures have been developed to induce an electric field gradient. The 2D microelectrodes of different geometries, such as polynomial, castellated, interdigitated, slanted and curved, are commonly patterned on the bottom surface of the microchannel. Alternatively, 3D microelectrodes are produced by fabricating planar electrodes on both top and bottom surfaces of the microchannel, extruded electrodes on the bottom, or vertical electrodes along the sidewalls of the channel.

\subsubsection{D planar microelectrodes}

Polynomial

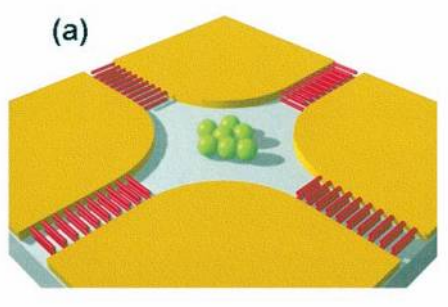

(b)

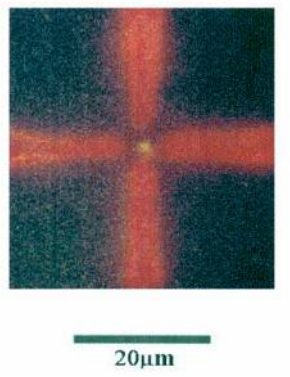

Figure 13 (a) Schematic illustration and (b) experimental image of the separation of TWV (red) and HSV (green) in a polynomial electrode [89]. Reprinted with permission from $\mathrm{H}$.

Morgan et al., Biophys. J. 77, 516 (1999). Copyright 1999 the Biophysical Society.

Yang et al [90] proposed a pair of triangular microelectrodes to manipulate and isolate the colorectal cancer HCT116 cells from the mixture with human embryonic kidney 293 HEK293 and E. coli cells, and similar electrode structure was applied by Alshareef et al [91] to isolate and separate human cancer cells. Morgan et al [89] achieved trapping and sorting of tobacco mosaic virus (TMV) and herpes simplex virus (HSV) type 1 in a polynomial electrode (Fig. 13). This design generates high electric fields along the edges of the electrode, and a low electric field at the center. Therefore, an HSV-1 experiencing negative DEP is trapped at the center of the electrode array, while TMV experiencing positive DEP is collected at the electrode edge. Asokan et al [92] utilized the electrode design to pattern actin 
on a substrate, and direct its motion on myosin substrates along the electric field lines. Also, trapping and concentration of DNA molecules were achieved under the combined effect of $\mathrm{AC}$ electroosmosis (ACEO), DEP and dipole-induced self-attraction [93], and the electrical properties of antibiotic-induced elongation of E. coli were characterized using similar quadruple electrode [94]. The patterning and collection of mammalian virus (HSV-1) by both positive and negative DEP at different frequencies was accomplished by Hughes et al [95] as well as the trapping of protein molecules [96] and bacterial spores [97] have been achieved in a triangular polynomial electrode.

Castellated

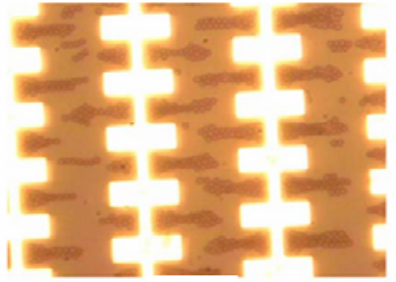

(a)

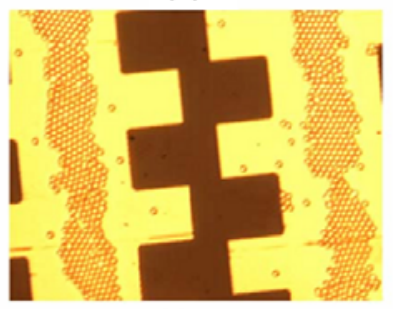

(c)

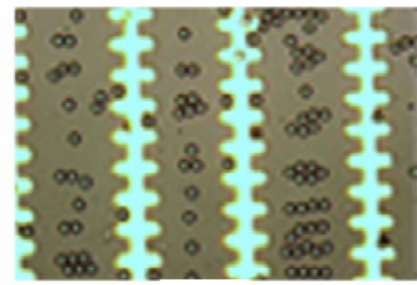

(b)

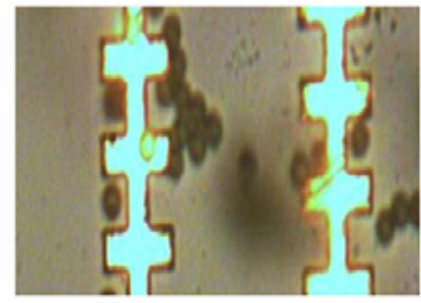

(d)

Figure 14 Experimental images of trapping of $5 \mu \mathrm{m}$ silica beads in a castellated electrode using (a-c) negative and (d) positive DEP [98]. Reprinted with permission from Q. Ramadan et al., Sensor Actuat. B-Chem. 113, 994 (2006). Copyright 2005 Elsevier.

One of the designs is castellated electrode arrays (Fig. 14), which generate an electric field gradient directly above their surfaces: high electric field regions located at the tips of the castellations, and low electric field regions in the bays between the tips. Morgan et al [89] utilized this design to separate submicro latex spheres by size, and separate carboxylatemodified and protein-functionalized latex sphere by surface charge. Other research work on trapping cells and silica beads [98], concentrating embryonic stem cells (ESCs) [99], sorting nano-[100] and micro-particles [101], clones of mouse melanoma B16F10 cells [102], separating cancel cells from blood [103,104], separating dormant Mycobacterium smegmatis from live and dead M. smegmatis [105] based on this design have also been reported. Choi et al [106] used this design to separate porcine oocytes based on the difference of 
dielectrophoretic velocity, which provides a new method for in vitro fertilization. By integrating the electrode array with a serpentine micropump and pneumatic microvalves, viable and non-viable cells were separated and collected, and nucleus was collected after cell lysis [107]. Ramadan et al [108] achieved lysing human white blood cells (WBC) and murine clonal cells (MN9D) in a continuous flow, and trapping silica beads that were selectively bonded to DNA at the same time. Zhang et al [109] utilized a similar structure with sharp tips to separate multi-walled carbon nanotubes (MWCNTs) and polystyrene microparticles according to their dielectric response. The MWCNTs exhibiting a positive DEP response were trapped at the regions of high electric fields, and were thus isolated from the polystyrene particles exhibiting a negative DEP response, which moved towards the regions of low electric fields. Yasukawa et al [110] optimized the design to rectangular features with different widths and gaps, and the manipulation and separation of microparticles depending on size has been demonstrated. Moreover, similar castellated microelectrode arrays were utilized by Suehiro [111] to dielectrophoretically fabricate and characterize nanomaterialbased sensors, such as a CNT gas sensor and a $\mathrm{ZnO}$ nanowire photosensor.

Interdigitated
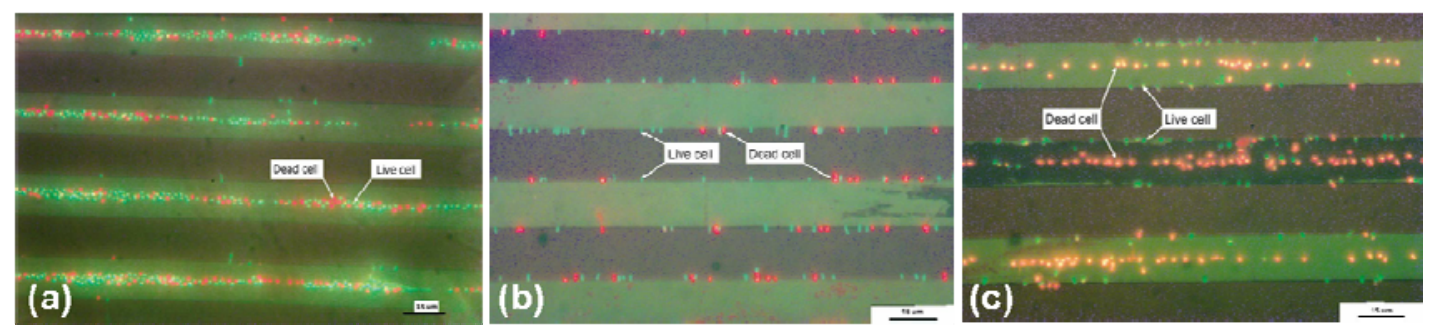

Figure 15 Experimental images of dielectrophoretic behaviours of live (green) and dead (red) cells on interdigitated microelectrode arrays: (a) positive DEP concentration of both $L$. innocua cells at the top centres of electrodes, (b) separation of Listeria cells, and (c) negative DEP concentration of both L. innocua cells at the edges of the electrodes [112]. Reprinted with permission from H. Li and R. Bashir, Sensor Actuat. B-Chem. 86, 215 (2002). Copyright 2002 Elsevier.

As shown in Fig. 15, interdigitated electrodes have been used to separate live and dead cells (i.e. Listeria innocua [112] and yeast cells [113]), and nano-/micro-particles [89,114], capture DNA [115], bacteria [36], polystyrene beads [114,116] and blood erythrocytes [114], capture and release ribosomal RNA (rRNA) [117], separate human colon cancer cells (HT-29 cells) from RBCs [118], characterize and separate C. parvum, C. muris, and G. lambia [119], and pattern colloid particles [120]. Park and Beskok [121] proposed a theoretical model to predict 
the AC electrokinetic motion of microparticles, which were validated by parametric experiments of colloid particles, including polystyrene and gold particles, and C. sporogenes bacterial spores, with interdigitated electrodes. Crews et al [122] developed an equation to calculate the electric gradient term above interdigitated electrodes, which is a function of the width and gaps of the electrode, and the applied voltage and the height above the electrode surface. The interdigitated electrodes have also integrated with other on-chip component for various applications. Suehiro et al $[123,124]$ proposed a method for detecting biological cells and bacteria called dielectrophoretic impedance measurement (DEPIM), which utilizes the positive DEP force to capture biological particles in suspension onto an interdigitated microelectrode array, and the transient variation of the electrical impedance to quantitatively evaluate the concentration of particles. The interdigitated electrodes also integrated with other on-chip components for various applications. Gadish and Voldman [116] developed a microconcentrator by combining interdigitated electrodes with a chaotic mixer, which achieved the concentration of particles (i.e. beads, spores and B. subtilis) with high throughput. Vykoukal et al [125] proposed a dielectrophoretic field-flow fractionation (DEPFFF) microseparator with a flex interdigitated electrode array to enrich stem cells from enzyme-digested adipose tissue, which was fabricated using a scalable and low-cost hybrid flex-circuit method. With the same DEP-FFF method, isolation of circulating tumor cells (CTCs) from blood was accomplished by Shim et al [126,127]. Lagally et al [128] developed an integrated microsystem consisting of an interdigitated electrode array, PDMS microvalves and an optical molecular beacon, which is capable of bacterial cell (i.e. E. coli) concentration and sequence-specific genetic detection.

Slanted

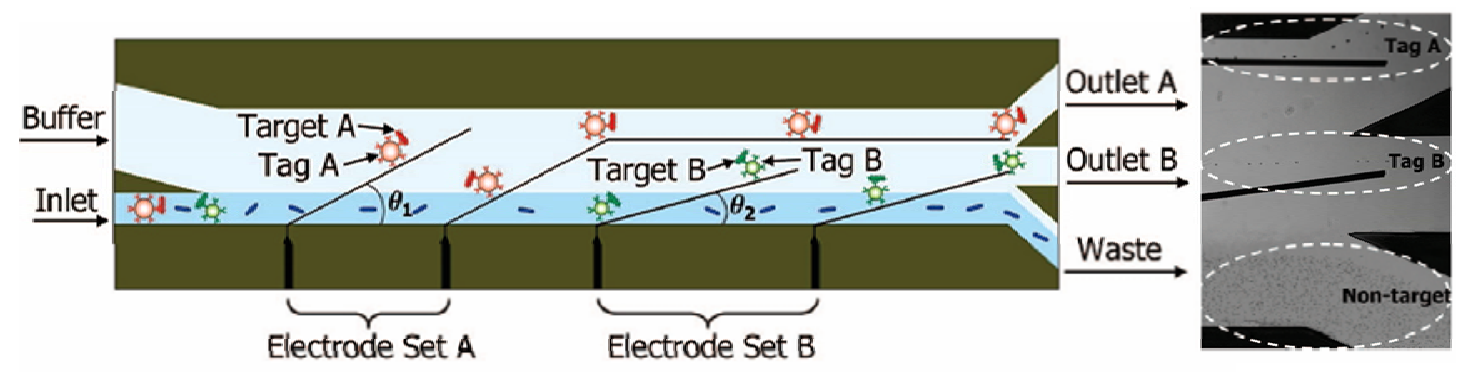

Figure 16 (a) Schematic illustration and (b) experimental image of multitarget bacterial cell separation in two sets of slanted electrode arrays [129]. Reprinted with permission from U. Kim et al., Anal. Chem. 80, 8656 (2008). Copyright 2008 American Chemical Society. 
In addition, slanted electrode arrays (i.e. electrodes placed at an angle to the direction of the flow, Fig. 16) have been utilized to separate particles in a continuous-flow manner. Kralj et al [130] used an array of slanted, interdigitated electrodes to separate polystyrene microspheres based on size. Larger particles are deflected more than the smaller particles due to the volume-dependent DEP force, different-sized particles hence are fractionated to distinct streams as they run down the length of the microchannel. By combining similar slanted electrode arrays with a diffusive mixer, Vahey and Voldman [131] achieved the separation of polystyrene particles based on surface conductance and the separation of live and dead yeast cells in an electrical conductivity gradient. Kuczenski et al [132] utilized pairs of microelectrodes at different angles to continuous flow to separate E. coli cells from RBCs. Kim et al [129] developed a microsorter with two sets of interdigitated electrode arrays placed at different angles to the direction of the flow to isolate and separate multiple target cells from mixtures into distinct fractions. Using a funnel-shaped electrode array, Pommer et al [133] demonstrated the separation of platelets from diluted whole blood. Han and Frazier [134] achieved the separation of red blood cells (RBCs) and white blood cells (WBCs) in both divergent and convergent interdigitated electrode arrays.

Curved
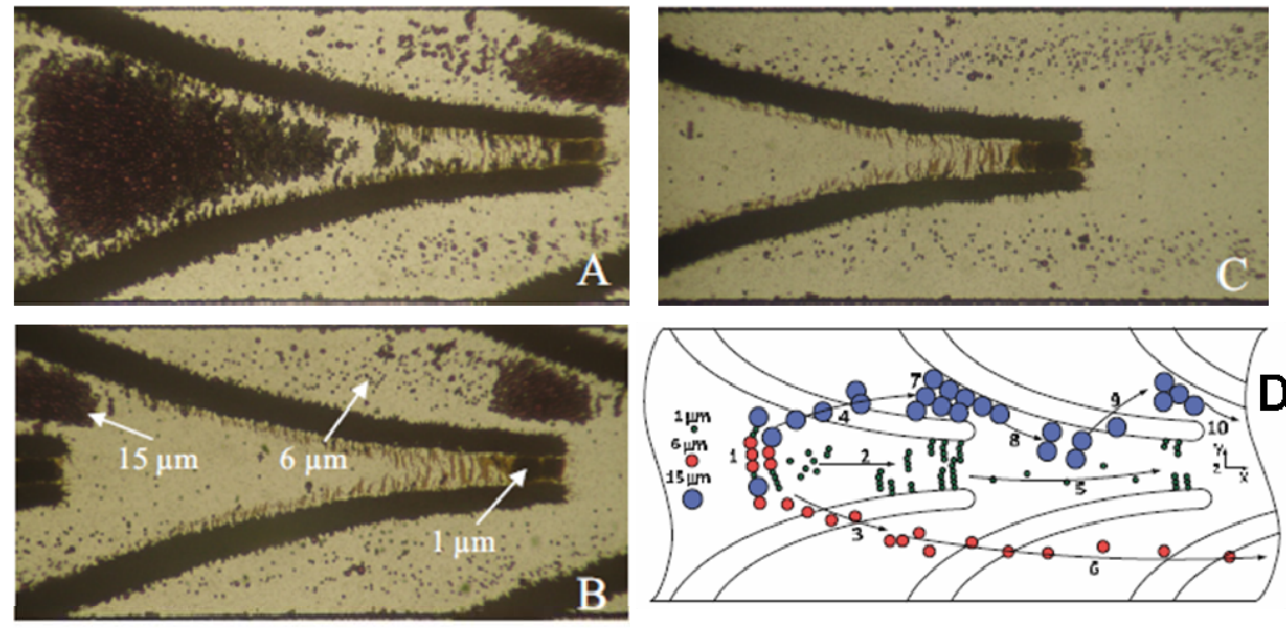

Figure 17 Experimental images (A-C) and schematic illustration (D) of sorting 1, 6 and 15 $\mu \mathrm{m}$ particles in curved electrode pairs [135]. Reprinted with permission from K.

Khoshmanesh et al., J. Appl. Phys. 108, 034904 (2010). Copyright 2010 American Institute of Physics.

Han et al [136] developed a piecewise curved electrode array placed at an angle to the direction of the flow to separate polystyrene particle according to size. Khoshmanesh et al 
developed a funnel-shaped curved electrode array (Fig. 17), which created a strong electric field gradient over the tips and maintains it over a large portion of the structure, and the induced electric field increase smoothly due to the curved structure. Based on this design, manipulation and separation of two-sized polystyrene particles [137], and the tunable multiplex separation of polystyrene particles at different frequencies [135] were demonstrated. By combining two sets of curved electrode arrays, the separation of live and dead yeast cells at different conductivities of suspending medium has been achieved, along with assessing the sorting performance on the downstream boomerang-shaped electrodes [138]. The authors also reported the patterning of multi-walled carbon nanotubes (MWCNTs) between curved microelectrodes to trap polystyrene particles with different surface modifications [139], and sort particles of different sizes [140]. The applied MWCNs having high conductivities can coat the surface of the microparticles, which induces their trapping at high frequencies, while also serving as a nanoelectrode extension of the microelectrode after the deposition. Circular microelectrode pairs were proposed by Jen and Chang [141] to generate a stepping electric field, and the concentration of HeLa cells was accomplished.

Other planar designs

Negative DEP microwells consisting of a square electrode and a line electrode have been utilized to pattern single particles, such as polystyrene beads of varies sizes [142], and adherent (HeLa) and non-adherent (HL60) cells [143]. Also, the capture of B. subtilis and C. sporogenes spores from high conductivity media was achieved by Koklu et al using negative DEP [144]. Jang et al [145] combined quadrupole and microwell electrodes for the manipulation and trapping of single particles (i.e. polystyrene microsphere and HeLa cell). The motion of the particle is manipulated by the alternating-current electrothermal effect (ACET) induced flow, and after the particle is positioned inside the capture range, the outer quadrupole electrodes responsible for manipulation are turned off while the microwell electrodes for trapping are actuated. Using an array (or matrix) of circular electrodes coated with porous hydrogel layers, Krishnan et al [146] separated polystyrene particles and DNAderivatized nanoparticles under high-conductive conditions. Moreover, the positioning of individual living cells (i.e. tumor cells [147] and yeast cells [148]) and droplets [148,149], and sorting individual live cells from an unlabelled population [150] have been achieved in integrated circuit chips with a grid pattern of electrode arrays. Ring-shaped electrodes proposed by Thomas et al $[151,152]$ were utilized to isolate, concentrate and separate microparticles and human osteoblast-like cells, and multiple concentric ring-shaped 
electrodes was applied to aggregate $M$. smegmatis [153], multilayered array and grid (MLAG) electrodes [154] composed of two metal layers and a sandwiched dielectric layer were employed to trap and pattern polystyrene particles using negative DEP, ring-dot-shaped electrodes were developed by Taff and Voldman [155] to trap and sort silver-coated beads and HeLa cells using positive DEP, also, the asymmetric electrode repeat patterns were utilized by Melvin et al [156] to collect microparticles and yeast cells from suspensions using combined ACEO and DEP effect. Another design involves differential coplanar electrode arrays, which has been utilized to capture and assemble green alga C. reinhardtii in fresh waters [157], and detect and actuate single Chinese hamster ovary (CHO) cells [158].

\subsubsection{D microelectrodes}

Compared to 2D planar electrodes that commonly patterned on the bottom of the microchannel, 3D electrodes, including the ones patterned on both top and bottom surface of the microchannel, extruded ones from microchannel bottom, the ones patterned on channel sidewalls, can increase the region where effective DEP effect taking place, allowing the improvement of microsystem efficiency. Also, it avoids the problem of particle adhesion on the surface of electrode or channel walls that occurred in dielectrophoretic devices with 2D electrodes.

Top-bottom patterned

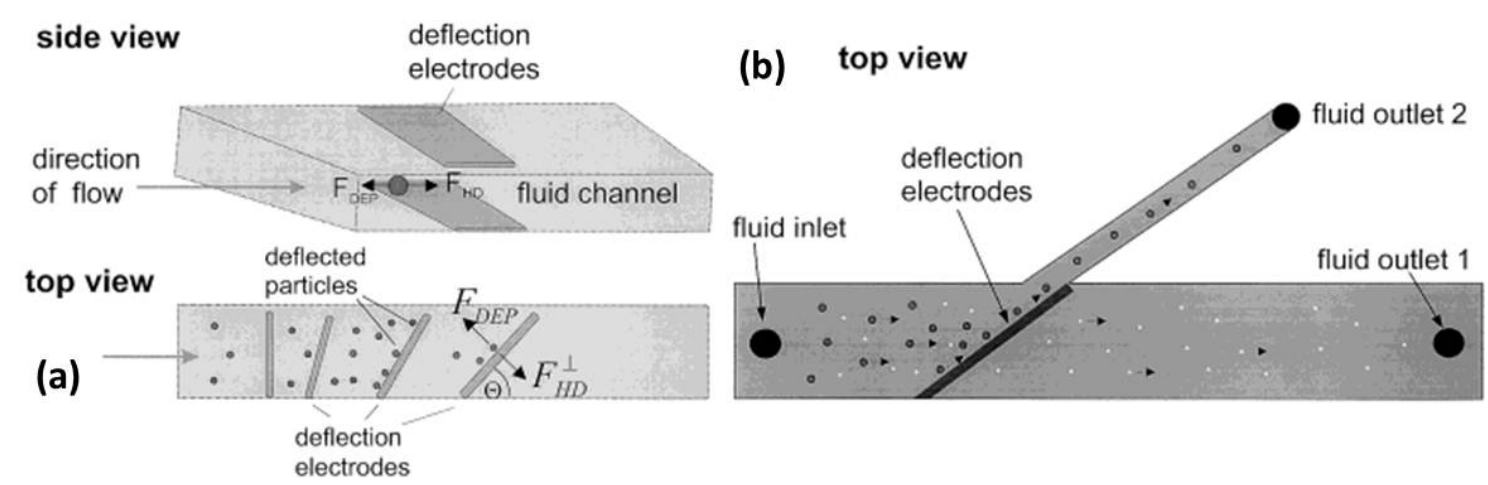

Figure 18 Top-bottom patterned microelectrode array [67] (a) schematic showing of design and mechanism, and (b) illustration of size-dependent particle separation. Reprinted with permission from M. Dürr et al., Electrophoresis 24, 722 (2003). Copyright 2003 WILEYVCH Verlag GmbH \& Co. KGaA, Weinheim.

A straightforward approach to overcome the limitations of 2D planar electrodes is fabricating planar microelectrodes on both the floor and ceiling of the microchannel, because the 
particles can be focused and forced to move round the centre of the channel in the height direction. Slanted electrode arrays have been patterned on both the top and bottom surfaces of the microchannel (Fig. 18) [67,68,70,71], which have been demonstrated for the manipulation, accumulation and separation of micro- and nanoparticles based on size. Yasukawa et al [159] used electrodes arrays consisting of navigator, cap and collector electrodes for immunoassays, which allows the manipulation and capture of polystyrene microparticles modified with anti-mouse IgG. Cheng et al [69] developed an integrated electrode array consisting of interdigitated, funnel-shaped, slanted and curved microelectodes, which could sort and concentrate heterogeneous populations including polystyrene microparticles, yeast cells and bacteria. Li et al [160] proposed an integrated array consisting of three units: a funnel-shaped focusing unit, a straight aligning unit, and a crescent-shaped trapping unit, which was used to concentrate different-sized polystyrene microparticles and yeast cells, separate polystyrene particles based on size, and separate $5 \mu \mathrm{m}$ polystyrene particles from yeast cells based on dielectric properties. Li et al [161] proposed using parallel multiple electrode arrays arranged in a radial direction for high-throughput performance, and captured polystyrene particle, yeast cells, and E-coli, and also separated live and yeast cells. Asymmetric microelectrode arrays which induce continuously varied electric fields along the transverse direction of a channel have been developed by Park et al [162], and have demonstrated their capability by sorting mouse P19 embryonic carcinoma (EC) and red blood cells (RBCs). Quadrupole electrodes have also been patterned on the top and bottom surface of the microchannel, and have managed to accumulate hepatitis A viruses [163], and capture and cultivate single yeast cell [164]. Yu et al [165] utilized a microelectrode array patterned on the circumference of an elliptic-like microchannel to focus microbeads and human leukemia HL60 cells towards the centre of the channel.

Extruded 


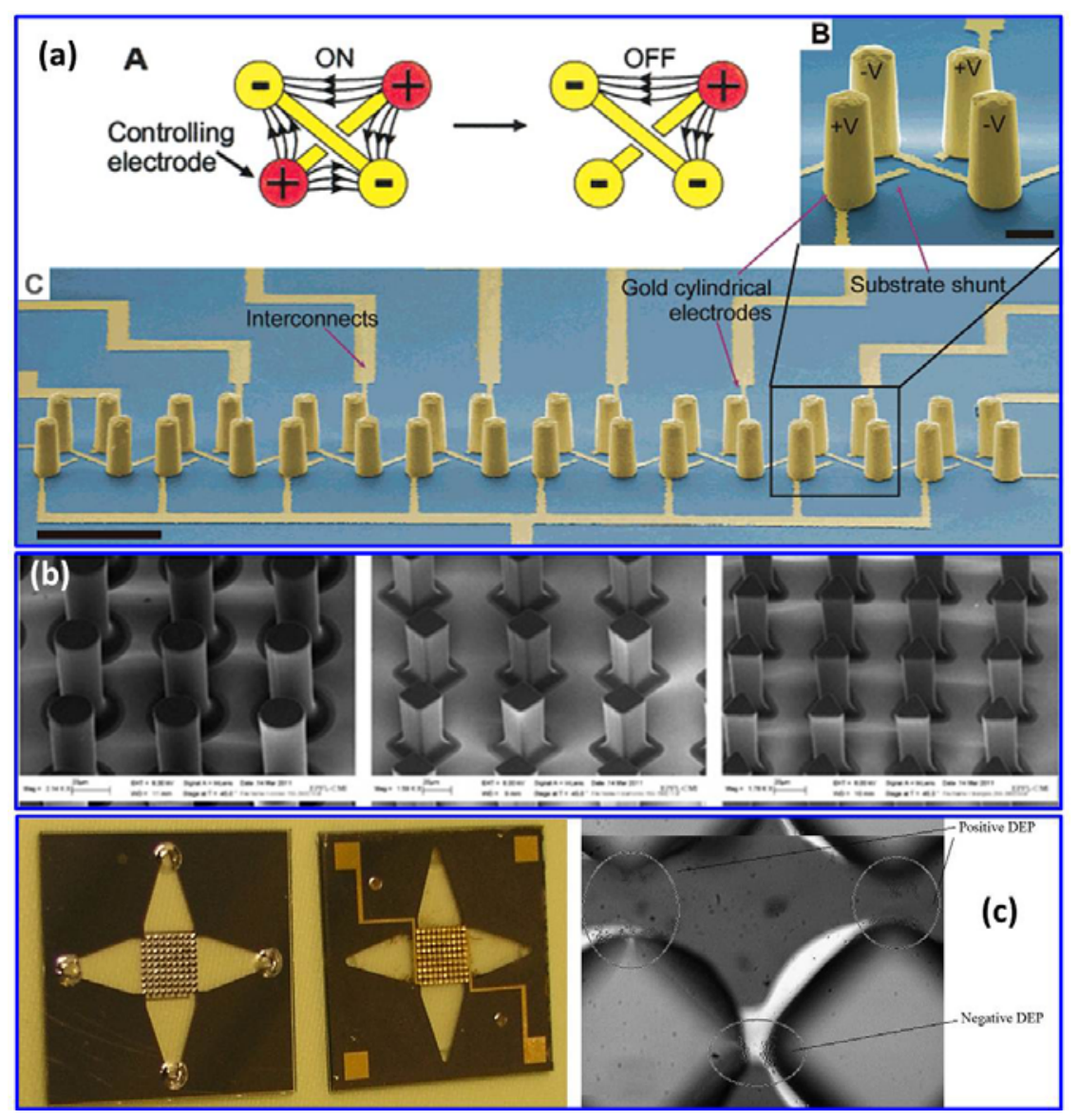

Figure 19 Extruded microelectrode structure. (a) Schematic illustration of the operating principle (A) and SEM images (B, C) of a single trap consisting of four gold cylindrical electrodes arranged trapezoidally [166]. Reprinted with permission from J. Voldman et al., Anal. Chem. 74, 3984 (2002). Copyright 2002 American Chemical Society. (b) SEM images of different-shaped 3D carbon microelectrode arrays [73]. Reprinted with permission from R.

Martinez-Duarte et al., Electrophoresis 32, 2385 (2011). Copyright 2011 WILEY-VCH

Verlag GmbH \& Co. KGaA, Weinheim. (c) left Images of DEP chips with highly doped

silicon pillar electrodes. right Experimental image of trapping cells using positive and negative DEP force [167]. Reprinted with permission from C. Iliescu et al., Sensor Actuat. BChem. 129, 491 (2008). Copyright 2007 Elsevier.

Another method to improve the volume where particles experience a strong DEP effect and the manipulation efficiency is fabricating extruded microelectrodes. Hunt et al [168] utilized an array of post-shaped gold electrodes to trap and manipulate a single polystyrene particle using positive and negative DEP, respectively. As shown in Fig. 19a, Voldman et al proposed the use of extruded gold posts in a quadrupolar arrangement fabricated by electroplating, and achieved the trapping of polystyrene microparticles [169] and HL60 cells [166]. Moreover, extruded 3D carbon (Fig. 19b) electrodes have been developed. Park and Madou [170] proposed using extruded carbon electrodes on the bottom substrate fabricated by carbonizing 
patterned SU-8 structures to improve the throughput. Using this structure, Jaramillo et al [171] trapped E. coli and separated E. coli from a mixture with B. cereus bacteria, while Duarte et al [172] trapped yeast cells from the mixture with polystyrene particles. Another 3D electrode structure is made from heavily doped silicon wafers, which simultaneously served as the walls of microfluidic channel (Fig. 19c). This 3D silicon electrode structure exhibits constant DEP forces along the height of the microfluidic channel, and achieves high trapping efficiency and high-throughput manipulation performance, which has been utilized to trap yeast cells [173], and separate live and dead yeast cells [167,174].

Sidewall patterned

(a)
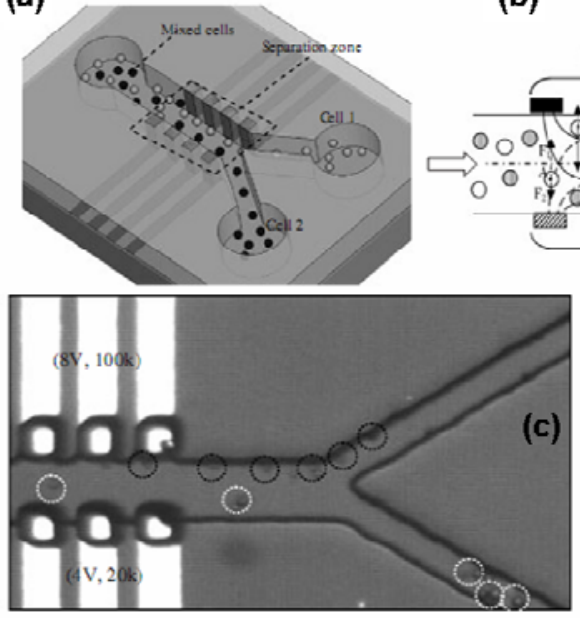

(b)
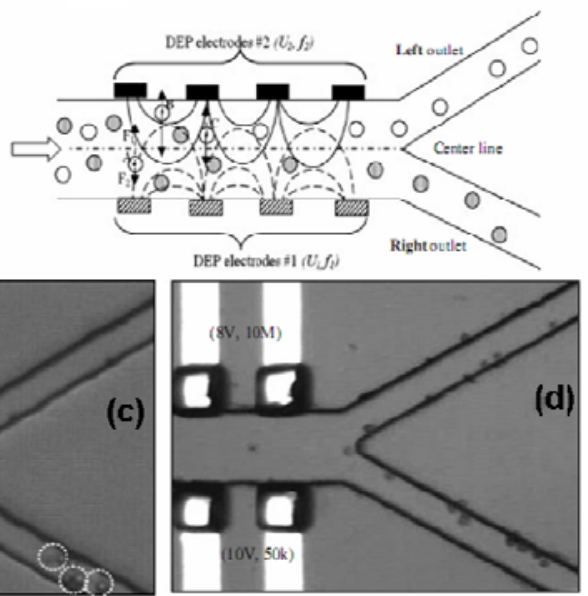

Figure 20 Microelectrodes arrays patterned along the microchannel sidewalls: (a) schematic of separation principle by an array of vertically interdigitated on both microchannel sidewalls, (b) schematic illustration of the balance of two DEP forces acting on cells in the separation zone, (c) experimental image of sorting HEK 293 (black circles) and N115 (white circles) cells, and (d) experiment image of sorting $6 \mu \mathrm{m}$ particles (right outlet) and HEK 293 cells (left outlet) [175]. Reprinted with permission from L. Wang et al., Electrophoresis 30, 782 (2009). Copyright 2010 WILEY-VCH Verlag GmbH \& Co. KGaA, Weinheim.

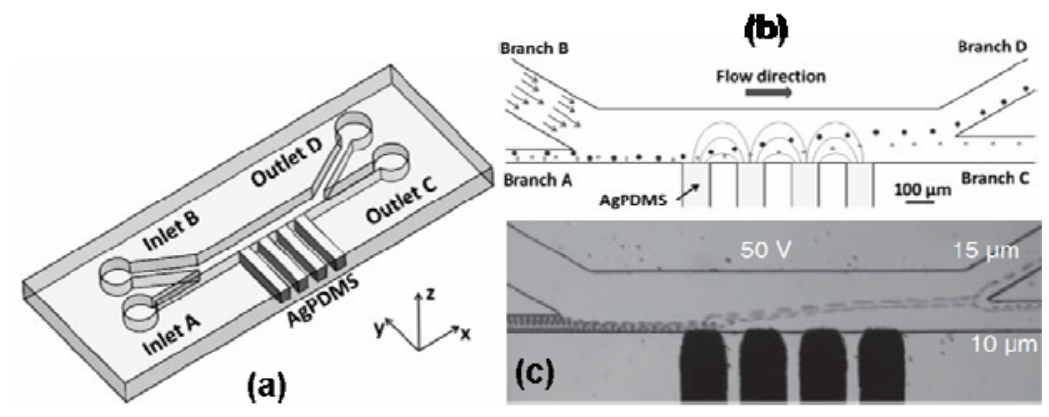


Figure 21 Conductive silver PDMS composite (AgPDMS) utilized as sidewall electrodes: (a) schematic of (a) device design and (b) size-dependent particle sorting principle, and (c) Experimental image showing the continuous separation of 10 and $15 \mu \mathrm{m}$ particles [80]. Reprinted with permission from N. Lewpiriyawong et al., Electrophoresis 31, 2622 (2010). Copyright 2010 WILEY-VCH Verlag GmbH \& Co. KGaA, Weinheim.
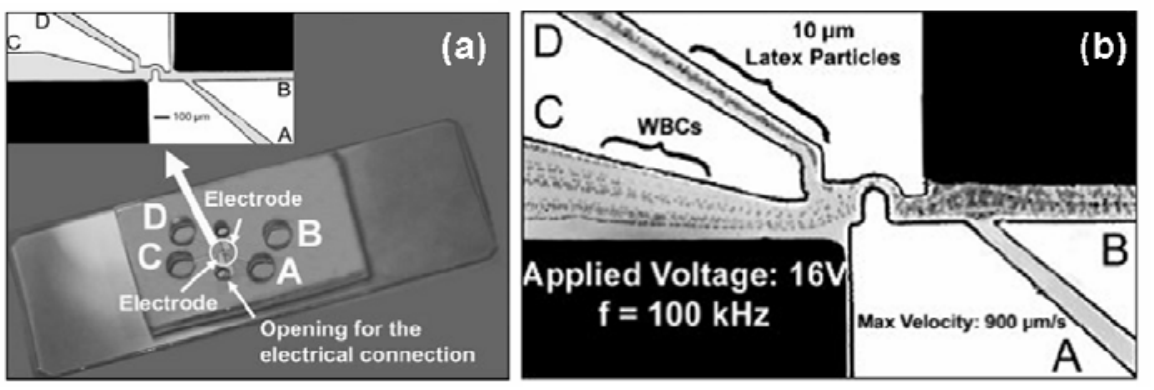

Figure 22 Copper microelectrodes patterned on microchannel sidewalls. (a) Picture of real fabricated DEP-based microdevices with an insulating hurdle and sidewall-patterned copper microelectrodes, and (b) experimental image showing the separation of WBCs and $10 \mu \mathrm{m}$ latex particles in the proposed design [176]. Reprinted with permission from B. Cetin and D. Li, Electrophoresis 31, 3035 (2010). Copyright 2010 WILEY-VCH Verlag GmbH \& Co.

KGaA, Weinheim.

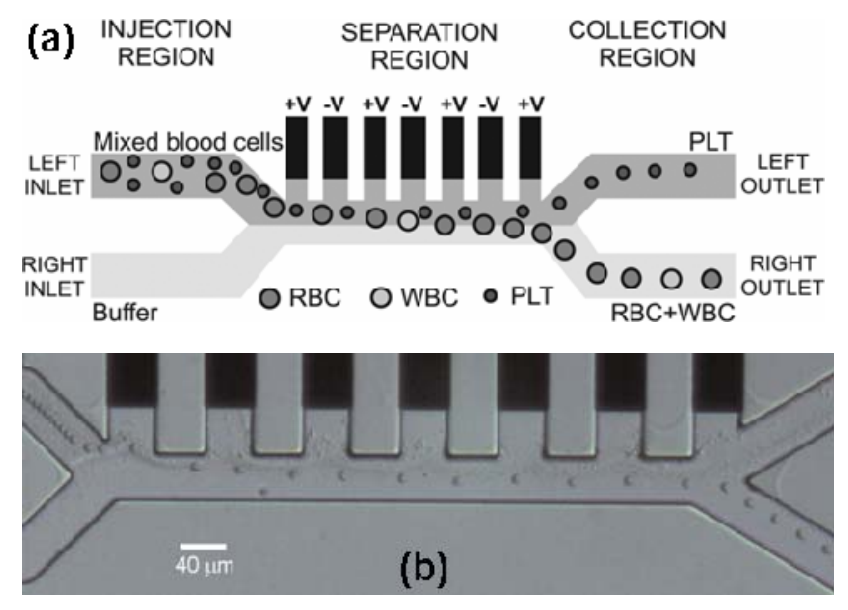

Figure 23 Liquid microelectrode array on the sidewall: (a) schematic of chip design and behaviour of RBCs, WBCs and PLTs under the DEP effect in the chip, and (b) experimental image showing the separation of RBCs and PLTs in the design [177]. Reprinted with permission from N. Piacentini et al., Biomicrofluidics 5, 034122 (2011). Copyright 2011 American institute of Physics.

Vertical microelectrode arrays have been also patterned along the sidewalls of the microchannel, which could extend the region for the DEP effect and induce nonuniform electric fields in the direction of channel width. Wang et al proposed using two vertical 
interdigitated electrode arrays embedded in both sidewalls of the microchannel (Fig. 20), and successfully switched polystyrene particles and mouse neural stem cells [178], and separated human kidney cells (HEK293) from N115 mouse neuroblastoma cells as well as polystyrene microbeads from modified HEK293 cells [175]. Lewpiriyawong et al developed a novel microfluidic device with conducting PDMS composites as sidewall electrodes (Fig. 21), and separated submicron and micron particles by size [80,179], yeast cells and E. coli from similar-size latex particles, and also separated live and dead yeast cells [180]. The conductive PDMS composite are made by mixing silver (Ag) powder with PDMS gel. By utilizing the combined effect of insulating hurdles and micro copper electrodes embedded along the sidewalls (Fig. 22), Kang et al [76] achieved the separation of mixtures of polystyrene particles having different sizes, and the separation of yeast cells with polystyrene particles. Cetin and $\mathrm{Li}$ [176] manipulated particles with different DEP responses and separated white blood cells (WBCs) from 10 um latex particles. Cetin et al [181] utilized two asymmetric copper electrodes embedded along the sidewalls for an electric field gradient, and then separated latex particles based on size and also separated yeast cells and white blood cells (WBCs). Microelectrode arrays wrapped around serpentine-shaped microchannel walls were utilized by $\mathrm{Hu}$ et al [182] to electrofuse human leukaemia K-562 cells. Moreover, distant and large metal electrodes (so-called liquid electrodes) patterned in access channels on the side of

the main channel (Fig. 23) have been utilized as a lateral electric gradient. Two arrays of liquid electrodes on both sides were used to focus polystyrene microparticles [78], sort viable and nonviable yeast cells [183], separate yeast cells in division [184], and separate the normal cells from the B. bovis infected red blood cells [184] and erythrocytes [185]. Moreover, liquid electrode arrays patterned on a single side were also utilized to deflect nano-/microparticles [186], and separate platelets from other blood cells [177].

\subsection{Insulator-based DEP devices}

As an alternative to DEP devices with microelectrode arrays patterned within the microchannels, insulating structures have also been employed to generate the required electric gradient, and electrodes are placed remotely at the end of the microchannel. Insulator-based DEP devices have several advantages over traditional electrode-based DEP devices, such as: (1) without the metal deposition process, injection moulding and hot embossing are relatively simple and applicable techniques for mass production, (2) as there are no electrodes embedded inside the channel, the device with insulating structures is robust 
and chemically inert, it has reduced surface fouling and no gas evaluation due to electrochemical reactions, and (3) particle motion taking advantage of the combined electrophoretic and electroomotic effect eliminates the need for additional hydrodynamic flow. There are two main insulating structures utilized to generate spatial electric field nonuniformities for the DEP effect: (1) single or multiple insulating obstacles embedded in microchannels, and (2) curved microchannels or channel geometry modification. In additional, contactless DEP devices have been developed where the electrode channels are isolated from the main channel by thin insulating barriers.

\subsubsection{Obstacles}

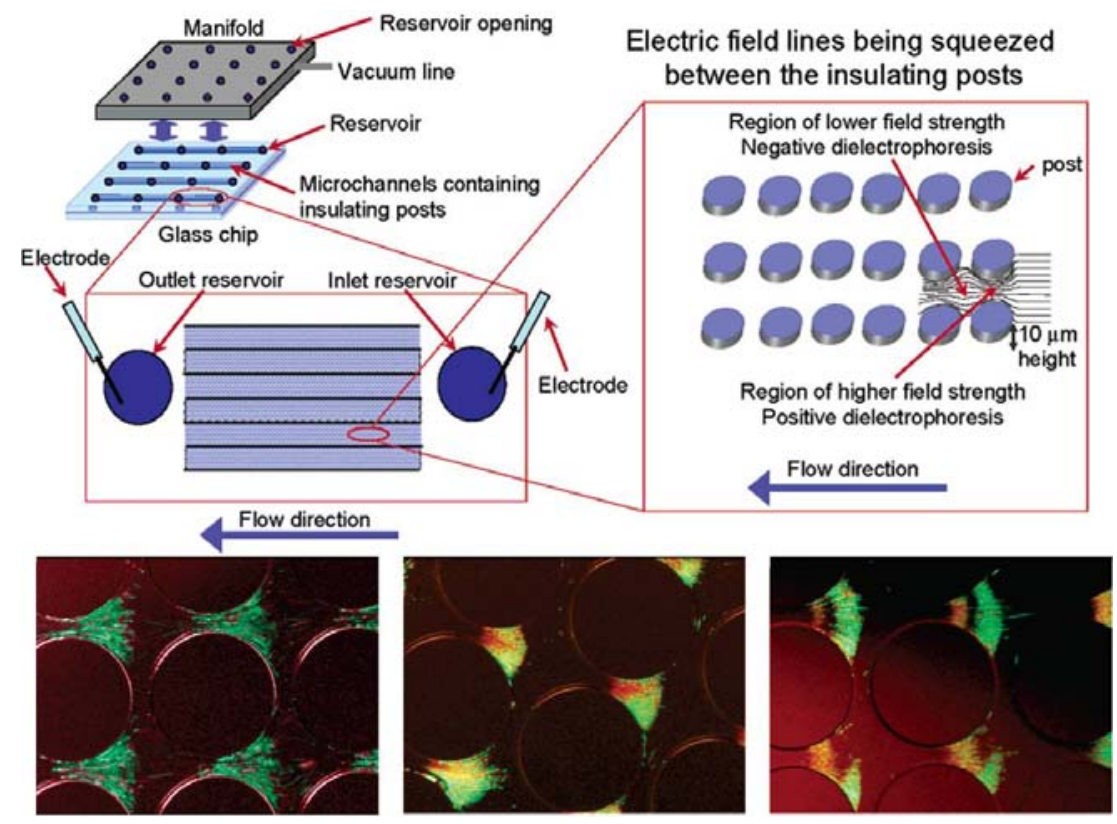

Figure 24 Insulating post arrays. top Schematic of device design, experimental setup and electric field lines constricted between insulating posts. bottom Experimental images showing the concentration and separation of live (green) and dead (red) E. coli in the chip [187]. Reprinted with permission from B. H. Lapizco-Encinas et al., Anal. Chem. 76, 1571 (2004). Copyright 2004 American Chemical Society.

Due to the dielectrophoretic effects induced in constricted regions between rows of posts, an array of circular or diamond-shaped insulating posts (Fig. 24) were embedded in the microchannels to manipulate a variety of particles, such as DNA $[188,189,190]$, protein [191,192], virus [193], live and dead bacteria [187,194]. It was demonstrated that the shape of the post and angle of the array with respect to applied electric field play an important role in the patterns of filaments and trapped zones [195], and the conductivity and $\mathrm{PH}$ value of the 
suspending medium can affect the performance [196]. Iliescu et al [197,198] used round insulating beads in a filter chip to trap particles. As the maximum electric field is generated at the points of contact between beads, particles experiencing a positive DEP force would be attracted near bead surface, while particles with a negative DEP would move to the gaps between the beads and then be flushed out with fluid flow.

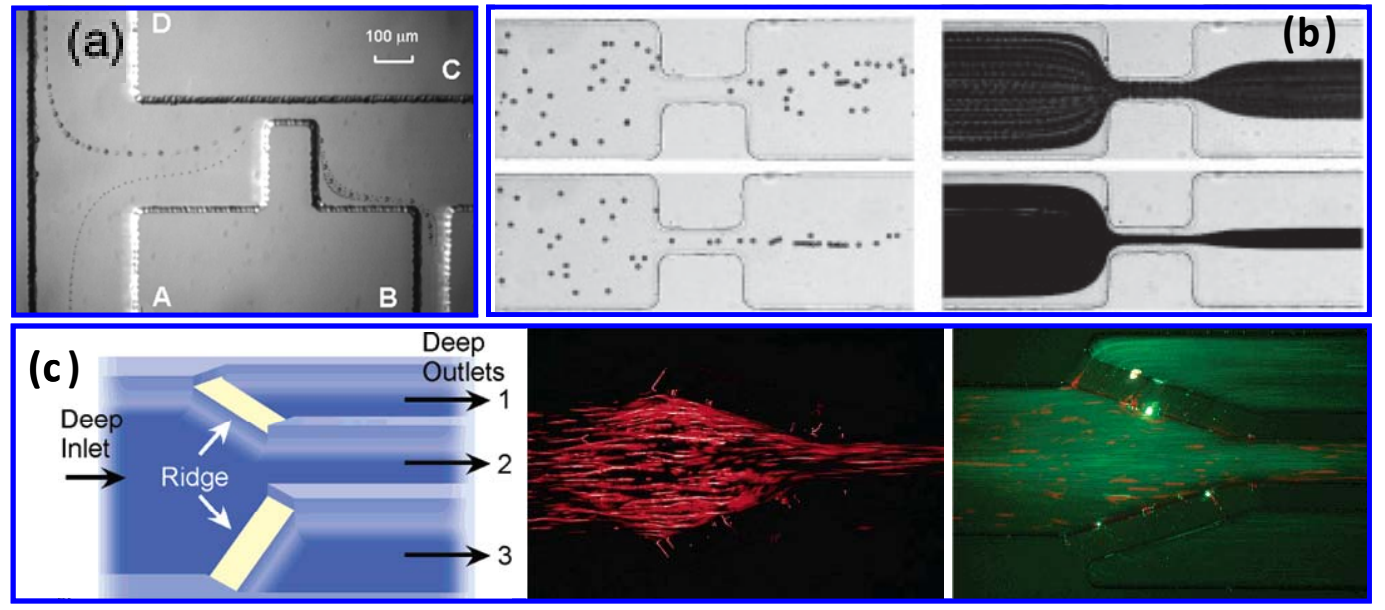

Figure 25 Other insulating obstacle-based structures. (a) Experimental image of sizedependent particle separation by a single rectangular hurdle [199]. Reprinted with permission

from K. H. Kang et al., Electrophoresis 27, 694 (2006). Copyright 2006 WILEY-VCH Verlag GmbH \& Co. KGaA, Weinheim. (b) Images (left snapshot, middle superimposed, right numerically predicted) of focusing microparticles by a pair of rectangular hurdles [84].

Reprinted with permission from J. Zhu and X. Xuan, Electrophoresis 30, 2668 (2009). Copyright 2010 WILEY-VCH Verlag GmbH \& Co. KGaA, Weinheim. (c) left Schematic of chip design with two ridges in the channel. middle Experimental image showing the concentration of $B$. subtilis. right Experimental image showing the separation of $B$. subtilis (red) and $20 \mathrm{~nm}$ particles (green) [200]. Reprinted with permission from L. M. Barrett et al., Anal. Chem. 77, 6798 (2007). Copyright 2005 American Chemical Society.

Rectangular and triangular obstacles have been used to sort and focus particles. Kang et al $[199,201]$ designed a rectangular insulating hurdle within a straight microchannel to separate particles by size (Fig. 25a). As the DEP force is proportional to the cube of the particle radius, particles of different sizes will experience different repulsive DEP forces at the hurdle of the hurdle, and hence can be deflected into distinct flow streams. Size-based separation of biological cells [202] and DNA molecules [203] was achieved using hurdles, and the triangular hurdle was developed to reduce the constriction gap region and its accompanying adverse effect on live cells. A pair of rectangular insulting hurdles was employed for focusing of polystyrene particles [84] (Fig. 25b), concentration and lysis of red blood cells, and separation of leukaemia cells from red blood cells [204]. Lewpiriyawong et al [205] utilized 
multiple rectangular insulating hurdles within an H-shaped microchannel for particle manipulation and contribution, which enhanced the DEP forces experienced by particles, and the controllability of particle motion. Instead of insulating solid obstacles, an oil droplet [206] and oil meniscus [88] were used to create spatial non-uniformities of electric field. By adjusting the size of the droplet, radius and penetration of the meniscus, the electric field gradients, and hence the induced DEP forces became easily controllable and adjustable to various separation configurations. Moreover, the insulating ridges [200,207] (Fig. 25c) in the depth of the microchannel were employed for spatial nonuniformities of the electric fields. As the motion of a particle depends on the ratio between the electrokinetic effect (the combined effect of electrophoresis and electroosmosis) and the DEP effect, such structures successfully trapped and deflected nano- [200] and micro-scale [207] polystyrene particles, and selectively concentrated and separated B. subtilis from 20-nm-diameter polystyrene particles [200]. Jen et al [208,209,210,211] utilized X-patterned insulating structures in the microchannel to squeeze the electric fields, which could generate a high field gradient at the constricted regions, when $\mathrm{AC}$ fields are applied via planer microelectrodes placed at the sides of the microchannel. These structures have demonstrated the focusing of polystyrene particles [208] and HeLa cells [209,211], and selective trapping of dead HeLa cells from mixed viable cells [210]. Recently, Patel et al [212] proposed the use of inherent electric field gradient at the reservoir-channel junction, and achieved the separation of live and dead yeast cells.

\subsubsection{Curved microchannels}

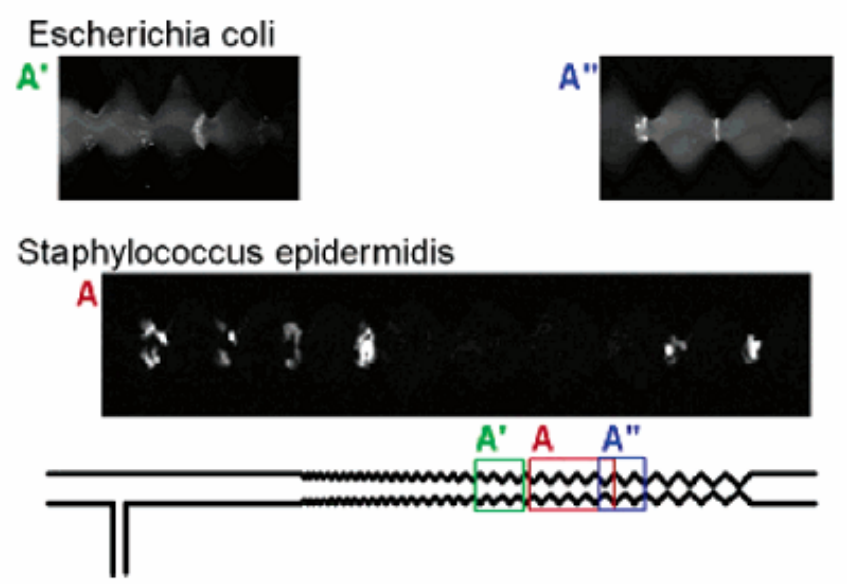

Figure 26 Separation of live and dead E. coli and S. epidermidis in a saw-tooth microchannel [213]. Reprinted with permission from M. D. Pysher and M. A. Hayes, Anal. Chem. 79, 4552 (2007). Copyright 2007 American Chemical Society. 
Besides embedding insulating obstacles in the straight microchannel, a variety of curved microchannels have also been used to manipulate particles. A saw-tooth channel (Fig. 26) was developed to separate bacteria (i.e. E. coli, B. subtilise and S. epidermidis) [213] and capture blood cells [214], which generates a series of gradually stronger field gradient along the length of the channnel. Chen et al [215] studied the particle movement in a nonconverging sawtooth channel, and the results indicated that particles can be selectively trapped by varying the channel geometry.

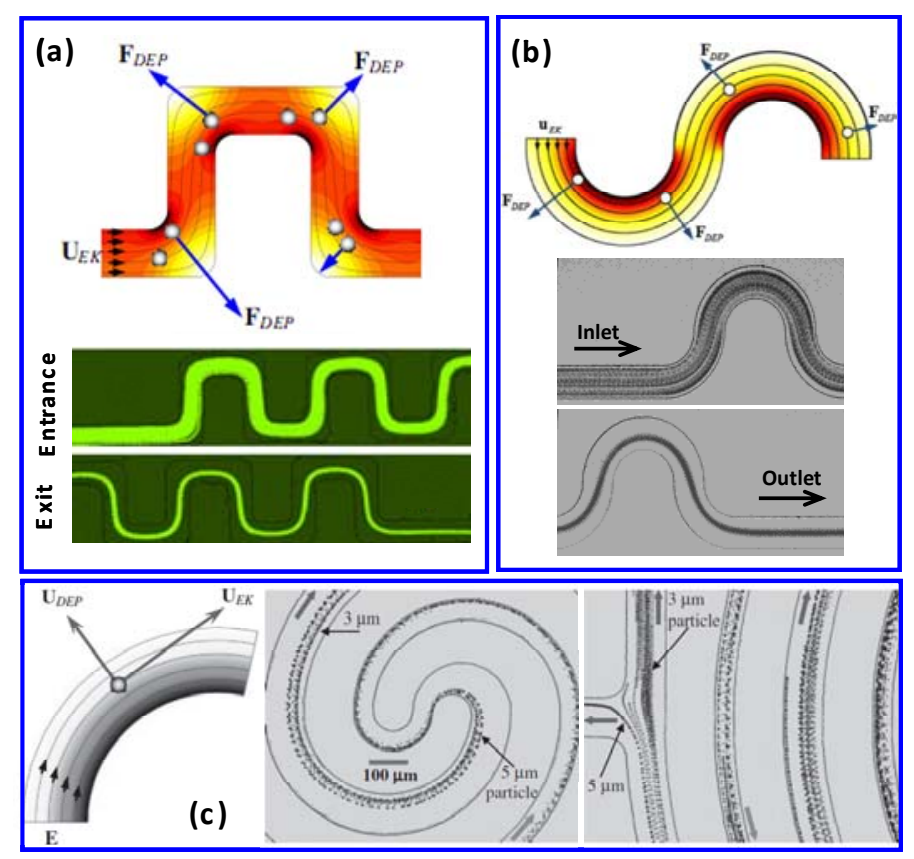

Figure 27 Channel geometry modification. (a) Schematic illustration of focusing principle in a serpentine microchannel using negative DEP (top), and experimental images showing the focusing behavior of $2.2 \mu \mathrm{m}$ particles by comparing the stream width at the entrance (middle) and exit (bottom) [216]. Reprinted with permission from C. Church et al., J. Micromech. Microeng. 20, 065011 (2010). Copyright 2010 IOP Publishing Ltd. (b) Schematic of particle focusing principle in a waved microchannel (top) and experimental images showing the focusing behavior of $15 \mu \mathrm{m}$ particles by comparing the stream width at inlet (middle) and outlet (bottom) [217]. Reprinted with permission from M. Li et al., J. Micromech. Microeng. 22, 095001 (2012). Copyright 2012 IOP Publishing Ltd. (c) Schematic illustration of particle motion in a spiral microchannel under the combined curvature-induced DEP and electrokinetic effects (left), and experimental images showing the sorting behavior of 3 and 5 $\mu \mathrm{m}$ particles at the centre (middle) and exit (right) region of the spiral channel [218]. Reprinted with permission from J. Zhu et al., Electrophoresis 31, 1382 (2010). Copyright 2003 WILEY-VCH Verlag GmbH \& Co. KGaA, Weinheim.

Xuan's group developed a serpentine channel (Fig. 27a), where the electric field becomes nonuniform at each U-turns with the maximum and minimum values obtained at the inner and outer corner, respectively. This design has been used to focus polystyrene particles 
[216,219] and cells [220], separate polystyrene particles based on size [221,222] and dielectrophoretic response [216], and separate yeast cells from E. coli [220] and polystyrene particles [221]. Li et al [217] proposed a waved microchannel comprising of 17 identical Sshaped curved microchannels in series (Fig. 27b), and focused polystyrene microparticles of varying sizes (i.e. 5, 10 and $15 \mu \mathrm{m}$ ) and yeast cells with different applied DC voltages. Moreover, Zhu et al developed spiral microchannels (Fig. 27c) to focus particles [223], and separate particles based on size [218] and charge [224]. Due to the variation of path length in the width of the channel, the applied electric field becomes uniform and attains maximum and minimum value near the inner and outer wall, respectively.

There were several other curved microchannel designs. Zhang et al [225] developed a circular channel to generate field gradients towards the circle centre, particles experiencing different DEP responses will be directed to different locations along the channel width direction, and thus be separated into different outlets. A series of circular microchannels [87] were utilized for particle concentration, which could focus the externally applied electric field stepwise: the field upstream is relatively lower and the electrokinetic effect responsible for continuously transporting particles is dominant, while the field downstream is higher and the dielectrophoretic effect responsible for trapping particles is dominant. The effects of obstacle and curvature were also combined to induce nonuniform electric fields. Li et al [226] proposed using an S-shaped microchannel embedded with multiple round hurdles to direct, position and focus fix-sized (i.e. 10 and $15 \mu \mathrm{m}$ ) polystyrene particles, and also separate them by size. Another novel design was presented by Cho et al [227], where microfabricated plastic membranes with honeycomb-typo pores were utilized to concentrate bacteria (i.e. $E$. coli). The bacteria could be captured at the edge of the pores when the applied AC field was turned to exhibit positive DEP effect.

\subsubsection{Contactless}




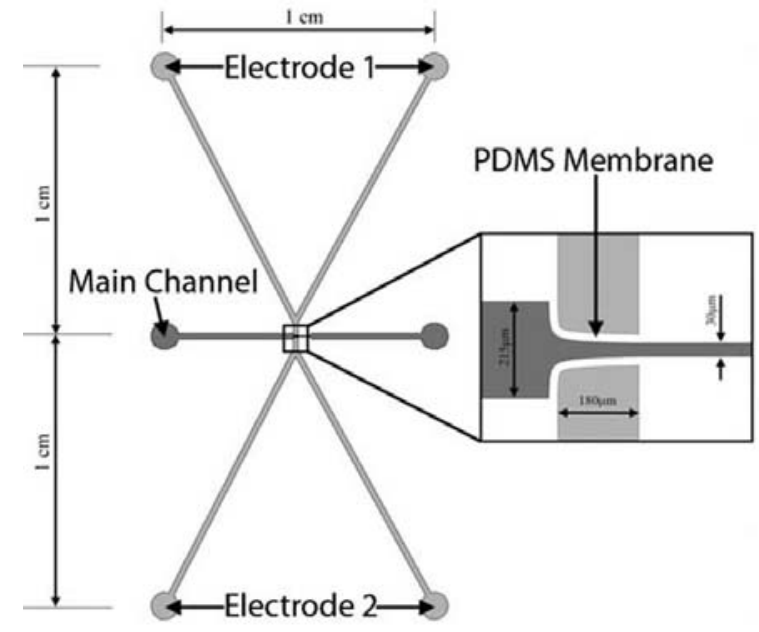

(a)

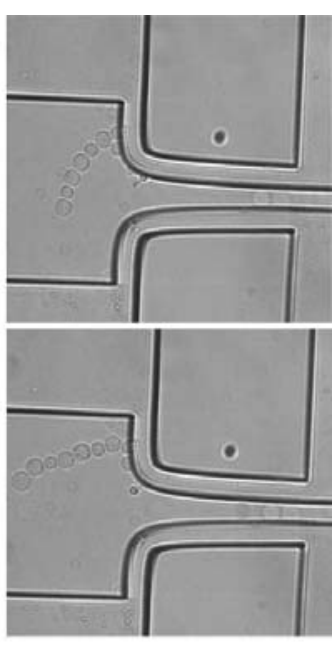

(b)

Figure 28 (a) Schematic illustration of contactless DEP microdevices design and configuration, and (b) experimental images showing cell chain formation by DEP effect [86].

Reprinted with permission from H. Shafiee et al., Biomed. Microdevices 11, 997 (2009). Copyright 2009 Springer.

Shafiee et al [86] developed a technique called contactless DEP (Fig. 28) to manipulate particles, where the electrodes are not in direct contact with the sample, but are inserted in a high conductive solution which is isolated from the main channel by thin insulating barriers. These insulating barriers exhibit a capacitive behavior, producing an electric field in the main channel when AC electric fields across the barriers are applied. This technique demonstrated the trapping of THP-1 human Leukemia monocytes and MCF-7 breast cancer cells [86], and also the sorting of live and dead THP-1 cells [228]. Moreover, this technique was utilized to selectively concentrate human breast cancer cells [229], isolate human leukemia cells from erythrocytes[230], separate tumor initiating cells (TICs) from non-TICs [231], and mouse ovarian surface epithelial (MOSE) cells from fibroblasts and macrophages [232], and characterize the electrical properties of different stages of MOSE cells [233].

\section{Conclusions and future perspectives}

In this review, we summarized the microfluidics-based techniques to manipulate and separate particles (i.e. mechanical, inertial, hydrodynamic, acoustic, optical, magnetic, and electrical technologies), in which dielectrophoresis is a label-free, non-destructive, inexpensive, highly sensitive and highly selective technique. Recent development in LOC or $\mu$ TAS results in different microfabrication techniques in dielectrophoretic applications, and enables the design of a large number of electrode- and insulator-based dielectrophoretic microdevices. 
Traditionally, 2D planar microelectrodes embedded within microchannels are fabricated by photolithography, thin-film deposition and lift-off techniques, while additional and more complicated techniques were used for 3D microelectrodes. For microelectrodes patterned on top and bottom surfaces of the microchannel, an essential step is bonding the two substrates with patterned microelectrodes, which can be achieved using adhesive and laser-patterned PDMS layer. The electroplating and pyrolysis techniques were used to build extruded microelectrodes, and heavily doped silicon wafers created by deep reactive ion etching (DRIE) technique can act as microelectrodes and microchannels simultaneously. Moreover, conductive silver PDMS composites, microscale copper, alloy microspheres were used as microelectrodes placed at the sidewalls of the microchannel. Compared to those with inchannel microelectrodes, the fabrication techniques for insulator-based DEP devices, such as soft lithography and etching, are relatively simple and cost-effective.

According to the approach used to induce spatial nonuniformities of electric fields for the DEP effect, developed dielectrophoretic microdevices could be classified into the devices with electrodes and insulators. Specifically, based on the configuration and structures of microelectrodes and insulators, the dielectrophoretic microdevices could be further classified into devices with 2D planar (i.e. polynomial, castellated, interdigitated, slanted curved and others), and 3D (i.e. top-bottom patterned, extruded, sidewall patterned) microelectrodes, devices with insulating obstacles (i.e. hurdle, oil droplet, oil meniscus and ridge) and curved microchannels (i.e. saw-tooth, serpentine, spiral, circular and waved), and contactless DEP devices.

Although the great advancement and achievement of fabrication techniques and structures of dielectrophoretic microdevices as reviewed in this work, further research are still required aimed to improve the performance DEP-based microsystems, and there are three main prospective aspects of future research work: fabrication, design and application.

(1) Fabrication techniques. DEP-based devices with microelectrodes embedded within microchannels offer several benefits. For example, Joule heating effect is avoided due to low operating voltages, moreover, the microdevice is more compatible with integrated circuits, and suitable for on-site analysis and characterization. However, the microfabrication of the interior metal microelectrodes requires relatively complex and expensive manufacturing 
techniques, such as thin-film deposition (i.e. evaporation and sputtering) and wet/dry etching. In addition, the fabrication procedures are not suitable for massive production. It seems that conductive polymer-based materials provide a solution to this issue, which could be used as electrodes and built in microfluidic devices via milling, drilling, and moulding techniques. Therefore, it makes great sense to invent alternative fabrication technologies for microelectrodes of varied materials rather than metal inside microchannels, which will enable new designs of intelligent and elaborate electrodes and insulating structures with more controllable localized electric fields, and inexpensive and mass fabrication.

(2) Device design and optimization. Although a variety of delectrophoretic microsytems have been developed in past decades, more studies regarding novel design or optimization need to be conducted. For electrode-based DEP microdevices, the structure and configuration of the microelectrodes determine the electric field distribution and hence DEP force. The development of integrated, intelligent, and advanced microelectrode arrays will achieve greater control of particle motion. In insulator-based DEP microdevices, as the applied field is confined within the nonconducting channel walls, and the local electric fields are defined by the morphology of the channel, optimizing or designing insulating microchannel structures with controllable localized electric fields is needed to increase the performance of DEP-based microsystems. Moreover, although the microdevices for DEP-based manipulation are simple, the instruments required for system operation are still bulky and complex, and the throughput of DEP-based is relatively low compared to traditional techniques. DEP-based microdevices could be integrated with different functionalities (i.e. detection, calculation, separation and concentration), and different components (i.e. pumps, mixers and valves) into a single all-inone chip to facilitate practical applications. Both electrode-and insulator-based DEP microdevices hence will be further developed towards the improvement of efficiency, throughput, integration and portability.

(3) Applications. It is of great use to extend the applications of proposed DEP-based microsystems to practical issues occurred in the field of biology, chemistry and medicine. However, polarization mechanisms for different bioparticles or chemicals must be understood, and extreme care must be taken to consider the proper DEP model and experimental conditions when applying DEP to biological systems and components. This is because these particles are varied greatly in shape, size, membrane and wall properties, organelle composition, each resulting in different electrical properties. In addition, the viability and 
functionality of bioparticles should not be affected after being exposure to electric fields. Several prospective applications of DEP technique involve: manipulation of micro- or nanoscale bioparticles such as cells, bacteria, viruses, protein and DNA, etc.; single-cell analysis (SCA) for investigating and elucidating cellular functions; conducting real-time and on-spot biochemical assays and reactions; and drug delivery. Therefore, the applications of proposed DEP-based microfluidic devices will be explored towards addressing needs in biological, chemical and medical fields.

\section{References}

[1] S. Haeberle, R. Zengerle, Microfluidic platforms for lab-on-a-chip applications, Lab on a Chip 7 (2007) 1094-1110.

[2] D. Mark, S. Haeberle, G. Roth, F. von Stetten, R. Zengerle, Microfluidic lab-on-a-chip platforms: requirements, characteristics and applications, Chemical Society Reviews 39 (2010) 1153-1182.

[3] D. Figeys, D. Pinto, Lab-on-a-chip: A revolution in biological and medical sciences, Analytical Chemistry 72 (2000) 330A-335A.

[4] Y.C. Lim, A.Z. Kouzani, W. Duan, Lab-on-a-chip: a component view, Microsystem Technologies-Microand Nanosystems-Information Storage and Processing Systems 16 (2010) 1995-2015.

[5] Y. Kang, D. Li, Electrokinetic motion of particles and cells in microchannels, Microfluidics and Nanofluidics 6 (2009) 431-460.

[6] B. Roda, A. Zattoni, P. Reschiglian, M.H. Moon, M. Mirasoli, E. Michelini, A. Roda, Field-flow fractionation in bioanalysis: A review of recent trends, Analytica Chimica Acta 635 (2009) 132-143.

[7] D.R. Gossett, W.M. Weaver, A.J. Mach, S.C. Hur, H.T.K. Tse, W. Lee, H. Amini, D. Di Carlo, Label-free cell separation and sorting in microfluidic systems, Analytical and Bioanalytical Chemistry 397 (2010) 3249-3267.

[8] P.R.C. Gascoyne, J. Vykoukal, Particle separation by dielectrophoresis, Electrophoresis 23 (2002) 19731983.

[9] M.P. Hughes, Strategies for dielectrophoretic separation in laboratory-on-a-chip systems, Electrophoresis 23 (2002) 2569-2582.

[10] H.A. Pohl, Dielectrophoresis : the behavior of neutral matter in nonuniform electric fields, Cambridge University Press, Cambridge, 1978.

[11] F.E.H. Tay, L. Yu, C. Iliescu, Particle Manipulation by Miniaturised Dielectrophoretic Devices, Defence Science Journal 59 (2009) 595-604.

[12] S. Fiedler, S.G. Shirley, T. Schnelle, G. Fuhr, Dielectrophoretic sorting of particles and cells in a microsystem, Analytical Chemistry 70 (1998) 1909-1915.

[13] C. Zhang, K. Khoshmanesh, A. Mitchell, K. Kalantar-zadeh, Dielectrophoresis for manipulation of micro/nano particles in microfluidic systems, Analytical and Bioanalytical Chemistry 396 (2010) 401420.

[14] B. Cetin, D. Li, Dielectrophoresis in microfluidics technology, Electrophoresis 32 (2011) 2410-2427.

[15] R. Pethig, Review Article-Dielectrophoresis: Status of the theory, technology, and applications, Biomicrofluidics 4 (2010).

[16] K. Khoshmanesh, S. Nahavandi, S. Baratchi, A. Mitchell, K. Kalantar-zadeh, Dielectrophoretic platforms for bio-microfluidic systems, Biosensors \& Bioelectronics 26 (2011) 1800-1814.

[17] Z.R. Gagnon, Cellular dielectrophoresis: Applications to the characterization, manipulation, separation and patterning of cells, Electrophoresis 32 (2011) 2466-2487.

[18] J. Voldman, Electrical forces for microscale cell manipulation, 2006, pp. 425-454.

[19] R. Martinez-Duarte, Microfabrication technologies in dielectrophoresis applications-A review, ELECTROPHORESIS 33 (2012) 3110-3132.

[20] J. Regtmeier, R. Eichhorn, M. Viefhues, L. Bogunovic, D. Anselmetti, Electrodeless dielectrophoresis for bioanalysis: Theory, devices and applications, Electrophoresis 32 (2011) 2253-2273.

[21] A.Y. Fu, C. Spence, A. Scherer, F.H. Arnold, S.R. Quake, A microfabricated fluorescence-activated cell sorter, Nat Biotech 17 (1999) 1109-1111. 
[22] S. Sergent-Tanguy, C. Chagneau, I. Neveu, P. Naveilhan, Fluorescent activated cell sorting (FACS): a rapid and reliable method to estimate the number of neurons in a mixed population, Journal of Neuroscience Methods 129 (2003) 73-79.

[23] A. Wolff, I.R. Perch-Nielsen, U.D. Larsen, P. Friis, G. Goranovic, C.R. Poulsen, J.P. Kutter, P. Telleman, Integrating advanced functionality in a microfabricated high-throughput fluorescent-activated cell sorter, Lab on a Chip 3 (2003) 22-27.

[24] S. Miltenyi, W. Muller, W. Weichel, A. Radbruch, HIGH-GRADIENT MAGNETIC CELLSEPARATION WITH MACS, Cytometry 11 (1990) 231-238.

[25] K. Smistrup, O. Hansen, H. Bruus, M.F. Hansen, Magnetic separation in microfluidic systems using microfabricated electromagnets-experiments and simulations, Journal of Magnetism and Magnetic Materials 293 (2005) 597-604.

[26] D.W. Inglis, R. Riehn, R.H. Austin, J.C. Sturm, Continuous microfluidic immunomagnetic cell separation, Applied Physics Letters 85 (2004) 5093-5095.

[27] W.W.-F. Leung, Centrifugal Separations in Biotechnology, Academic Press, Oxford, 2007.

[28] E. Sollier, H. Rostaing, P. Pouteau, Y. Fouillet, J.-L. Achard, Passive microfluidic devices for plasma extraction from whole human blood, Sensors and Actuators B: Chemical 141 (2009) 617-624.

[29] P. Sethu, A. Sin, M. Toner, Microfluidic diffusive filter for apheresis (leukapheresis), Lab on a Chip 6 (2006) 83-89.

[30] V. VanDelinder, A. Groisman, Perfusion in microfluidic cross-flow: Separation of white blood cells from whole blood and exchange of medium in a continuous flow, Analytical Chemistry 79 (2007) 20232030.

[31] V. VanDelinder, A. Groisman, Separation of plasma from whole human blood in a continuous cross-flow in a molded microfluidic device, Analytical Chemistry 78 (2006) 3765-3771.

[32] X. Chen, D. Cui, L. Zhang, Isolation of plasma from whole blood using a microfludic chip in a continuous cross-flow, Chinese Science Bulletin 54 (2009) 324-327.

[33] S. Zheng, H. Lin, J.-Q. Liu, M. Balic, R. Datar, R.J. Cote, Y.-C. Tai, Membrane microfilter device for selective capture, electrolysis and genomic analysis of human circulating tumor cells, Journal of Chromatography A 1162 (2007) 154-161.

[34] P. Wilding, L.J. Kricka, J. Cheng, G. Hvichia, M.A. Shoffner, P. Fortina, Integrated cell isolation and polymerase chain reaction analysis using silicon microfilter chambers, Analytical Biochemistry 257 (1998) 95-100.

[35] H. Mohamed, J.N. Turner, M. Caggana, Biochip for separating fetal cells from maternal circulation, Journal of Chromatography A 1162 (2007) 187-192.

[36] L.J. Yang, P.P. Banada, M.R. Chatni, K.S. Lim, A.K. Bhunia, M. Ladisch, R. Bashir, A multifunctional micro-fluidic system for dielectrophoretic concentration coupled with immuno-capture of low numbers of Listeria monocytogenes, Lab on a Chip 6 (2006) 896-905.

[37] D.R. Gossett, D.D. Carlo, Particle Focusing Mechanisms in Curving Confined Flows, Analytical Chemistry 81 (2009) 8459-8465.

[38] D. Di Carlo, D. Irimia, R.G. Tompkins, M. Toner, Continuous inertial focusing, ordering, and separation of particles in microchannels, Proceedings of the National Academy of Sciences of the United States of America 104 (2007) 18892-18897.

[39] D. Di Carlo, J.F. Edd, D. Irimia, R.G. Tompkins, M. Toner, Equilibrium separation and filtration of particles using differential inertial focusing, Analytical Chemistry 80 (2008) 2204-2211.

[40] S.S. Kuntaegowdanahalli, A.A.S. Bhagat, G. Kumar, I. Papautsky, Inertial microfluidics for continuous particle separation in spiral microchannels, Lab on a Chip 9 (2009) 2973-2980.

[41] A.J. Mach, D. Di Carlo, Continuous Scalable Blood Filtration Device Using Inertial Microfluidics, Biotechnology and Bioengineering 107 (2010) 302-311.

[42] J.-S. Park, S.-H. Song, H.-I. Jung, Continuous focusing of microparticles using inertial lift force and vorticity via multi-orifice microfluidic channels, Lab on a Chip 9 (2009) 939-948.

[43] M. Yamada, M. Seki, Hydrodynamic filtration for on-chip particle concentration and classification utilizing microfluidics, Lab on a Chip 5 (2005) 1233-1239.

[44] M. Yamada, M. Nakashima, M. Seki, Pinched flow fractionation: Continuous size separation of particles utilizing a laminar flow profile in a pinched microchannel, Analytical Chemistry 76 (2004) 5465-5471.

[45] L.R. Huang, E.C. Cox, R.H. Austin, J.C. Sturm, Continuous particle separation through deterministic lateral displacement, Science 304 (2004) 987-990.

[46] S. Zheng, Y.-C. Tai, H. Kasdan, Ieee, A micro device for separation of erythrocytes and leukocytes in human blood, 2005 27th Annual International Conference of the IEEE Engineering in Medicine and Biology Society, Vols 1-7, 2005, pp. 1024-1027. 
[47] J.A. Davis, D.W. Inglis, K.J. Morton, D.A. Lawrence, L.R. Huang, S.Y. Chou, J.C. Sturm, R.H. Austin, Deterministic hydrodynamics: Taking blood apart, Proceedings of the National Academy of Sciences of the United States of America 103 (2006) 14779-14784.

[48] D.W. Inglis, J.A. Davis, T.J. Zieziulewicz, D.A. Lawrence, R.H. Austin, J.C. Sturm, Determining blood cell size using microfluidic hydrodynamics, Journal of Immunological Methods 329 (2008) 151-156.

[49] F. Petersson, A. Nilsson, C. Holm, H. Jonsson, T. Laurell, Separation of lipids from blood utilizing ultrasonic standing waves in microfluidic channels, Analyst 129 (2004) 938-943.

[50] F. Petersson, L. Aberg, A.-M. Sward-Nilsson, T. Laurell, Free flow acoustophoresis: Microfluidic-based mode of particle and cell separation, Analytical Chemistry 79 (2007) 5117-5123.

[51] S. Kapishnikov, V. Kantsler, V. Steinberg, Continuous particle size separation and size sorting using ultrasound in a microchannel, Journal of Statistical Mechanics-Theory and Experiment (2006).

[52] J.J. Hawkes, W.T. Coakley, Force field particle filter, combining ultrasound standing waves and laminar flow, Sensors and Actuators B-Chemical 75 (2001) 213-222.

[53] A. Ashkin, J.M. Dziedzic, OPTICAL TRAPPING AND MANIPULATION OF SINGLE LIVING CELLS USING INFRARED-LASER BEAMS, Berichte Der Bunsen-Gesellschaft-Physical Chemistry Chemical Physics 93 (1989) 254-260.

[54] A. Ashkin, J.M. Dziedzic, T. Yamane, OPTICAL TRAPPING AND MANIPULATION OF SINGLE CELLS USING INFRARED-LASER BEAMS, Nature 330 (1987) 769-771.

[55] W.H. Wright, G.J. Sonek, Y. Tadir, M.W. Berns, LASER TRAPPING IN CELL BIOLOGY, Ieee Journal of Quantum Electronics 26 (1990) 2148-2157.

[56] J. Jung, K.-H. Han, Lateral-driven continuous magnetophoretic separation of blood cells, Applied Physics Letters 93 (2008).

[57] K.H. Han, A.B. Frazier, Continuous magnetophoretic separation of blood cells in microdevice format, Journal of Applied Physics 96 (2004) 5797-5802.

[58] K.H. Han, A.B. Frazier, Diamagnetic capture mode magnetophoretic microseparator for blood cells, Journal of Microelectromechanical Systems 14 (2005) 1422-1431.

[59] N. Pamme, C. Wilhelm, Continuous sorting of magnetic cells via on-chip free-flow magnetophoresis, Lab on a Chip 6 (2006) 974-980.

[60] W. Korohoda, A. Wilk, Cell electrophoresis - A method for cell separation and research into cell surface properties, Cellular \& Molecular Biology Letters 13 (2008) 312-326.

[61] T.B. Jones, Electromechanics of Particles, Cambridge Unviersity Press, Cambridge, 1995.

[62] H. Morgan, N.G. Green, AC Electrokinetic: Colloids and Nanoparticles, Research Studies Press, London, 2003.

[63] P.R.C. Gascoyne, J.V. Vykoukal, Dielectrophoresis-based sample handling in general-purpose programmable diagnostic instruments, Proceedings of the Ieee 92 (2004) 22-42.

[64] M.P. Hughes, R. Pethig, X.B. Wang, Dielectrophoretic forces on particles in travelling electric fields, Journal of Physics D-Applied Physics 29 (1996) 474-482.

[65] M. Dimaki, P. Boggild, Dielectrophoresis of carbon nanotubes using microelectrodes: a numerical study, Nanotechnology 15 (2004) 1095-1102.

[66] M. Li, S. Li, J. Wu, W. Wen, W. Li, G. Alici, A simple and cost-effective method for fabrication of integrated electronic-microfluidic devices using a laser-patterned PDMS layer, Microfluidics and Nanofluidics 12 (2012) 751-760.

[67] M. Durr, J. Kentsch, T. Muller, T. Schnelle, M. Stelzle, Microdevices for manipulation and accumulation of micro- and nanoparticles by dielectrophoresis, Electrophoresis 24 (2003) 722-731.

[68] J. Kentsch, M. Durr, T. Schnelle, G. Gradl, T. Muller, M. Jager, A. Normann, M. Stelzle, Microdevices for separation, accumulation, and analysis of biological micro- and nanoparticles, IEE proceedings. Nanobiotechnology 150 (2003) 82-89.

[69] I.F. Cheng, H.-C. Chang, D. Hou, H.-C. Chang, An integrated dielectrophoretic chip for continuous bioparticle filtering, focusing, sorting, trapping, and detecting, Biomicrofluidics 1 (2007).

[70] D.F. Chen, H. Du, W.H. Li, A 3D paired microelectrode array for accumulation and separation of microparticles, Journal of Micromechanics and Microengineering 16 (2006) 1162-1169.

[71] D. Chen, H. Du, A dielectrophoretic barrier-based microsystem for separation of microparticles, Microfluidics and Nanofluidics 3 (2007) 603-610.

[72] J. Voldman, M. Toner, M.L. Gray, M.A. Schmidt, A dielectrophoresis-based array cytometer, 2001.

[73] R. Martinez-Duarte, P. Renaud, M.J. Madou, A novel approach to dielectrophoresis using carbon electrodes, Electrophoresis 32 (2011) 2385-2392.

[74] C. Iliescu, G.L. Xu, V. Samper, F.E.H. Tay, Fabrication of a dielectrophoretic chip with 3D silicon electrodes, Journal of Micromechanics and Microengineering 15 (2005) 494-500.

[75] L. Wang, L. Flanagan, A.P. Lee, Side-wall vertical electrodes for lateral field microfluidic applications, Journal of Microelectromechanical Systems 16 (2007) 454-461. 
[76] Y. Kang, B. Cetin, Z. Wu, D. Li, Continuous particle separation with localized AC-dielectrophoresis using embedded electrodes and an insulating hurdle, Electrochimica Acta 54 (2009) 1715-1720.

[77] S. Li, M. Li, Y.S. Hui, W. Cao, W. Li, W. Wen, A novel method to construct 3D electrodes at the sidewall of microfluidic channel, Microfluidics and Nanofluidics 14 (2013) 499-508.

[78] N. Demierre, T. Braschler, P. Linderholm, U. Seger, H. van Lintel, P. Renaud, Characterization and optimization of liquid electrodes for lateral dielectrophoresis, Lab on a Chip 7 (2007) 355-365.

[79] J. Luo, E. Nelson, G.P. Li, M. Bachman, Electronic-microfluidic system for sorting particles and whole blood using gel electrodes, Electronic Components and Technology Conference (ECTC), 2013 IEEE 63rd, 2013, pp. 1905-1911.

[80] N. Lewpiriyawong, C. Yang, Y.C. Lam, Continuous sorting and separation of microparticles by size using AC dielectrophoresis in a PDMS microfluidic device with 3-D conducting PDMS composite electrodes, Electrophoresis 31 (2010) 2622-2631.

[81] Y.N. Xia, G.M. Whitesides, Soft lithography, Annual Review of Materials Science 28 (1998) 153-184.

[82] G.M. Whitesides, A.D. Stroock, Flexible methods for microfluidics, Physics Today 54 (2001) 42-48.

[83] G.M. Whitesides, The origins and the future of microfluidics, Nature 442 (2006) 368-373.

[84] J. Zhu, X. Xuan, Dielectrophoretic focusing of particles in a microchannel constriction using DC-biased AC flectric fields, Electrophoresis 30 (2009) 2668-2675.

[85] J.C. McDonald, D.C. Duffy, J.R. Anderson, D.T. Chiu, H.K. Wu, O.J.A. Schueller, G.M. Whitesides, Fabrication of microfluidic systems in poly(dimethylsiloxane), Electrophoresis 21 (2000) 27-40.

[86] H. Shafiee, J.L. Caldwell, M.B. Sano, R.V. Davalos, Contactless dielectrophoresis: a new technique for cell manipulation, Biomedical Microdevices 11 (2009) 997-1006.

[87] D. Chen, H. Du, A microfluidic device for rapid concentration of particles in continuous flow by DC dielectrophoresis, Microfluidics and Nanofluidics 9 (2010) 281-291.

[88] P.K. Thwar, J.J. Linderman, M.A. Burns, Electrodeless direct current dielectrophoresis reconfigurable field-shaping oil barriers using, Electrophoresis 28 (2007) 4572-4581.

[89] H. Morgan, M.P. Hughes, N.G. Green, Separation of submicron bioparticles by dielectrophoresis, Biophysical Journal 77 (1999) 516-525.

[90] F. Yang, X. Yang, H. Jiang, P. Bulkhaults, P. Wood, W. Hrushesky, G. Wang, Dielectrophoretic separation of colorectal cancer cells, Biomicrofluidics 4 (2010).

[91] M. Alshareef, N. Metrakos, E. Juarez Perez, F. Azer, F. Yang, X. Yang, G. Wang, Separation of tumor cells with dielectrophoresis-based microfluidic chip, Biomicrofluidics 7 (2013).

[92] S.B. Asokan, L. Jawerth, R.L. Carroll, R.E. Cheney, S. Washburn, R. Superfine, Two-dimensional manipulation and orientation of actin-myosin systems with dielectrophoresis, Nano Letters 3 (2003) 431-437.

[93] J.R. Du, Y.J. Juang, J.T. Wu, H.H. Wei, Long-range and superfast trapping of DNA molecules in an ac electrokinetic funnel, Biomicrofluidics 2 (2008).

[94] C. Chung, I. Cheng, W. Yang, H. Chang, Antibiotic susceptibility test based on the dielectrophoretic behavior of elongated Escherichia coli with cephalexin treatment, Biomicrofluidics 5 (2011).

[95] M.P. Hughes, H. Morgan, F.J. Rixon, J.P.H. Burt, R. Pethig, Manipulation of herpes simplex virus type 1 by dielectrophoresis, Biochimica Et Biophysica Acta-General Subjects 1425 (1998) 119-126.

[96] R. Hölzel, N. Calander, Z. Chiragwandi, M. Willander, F.F. Bier, Trapping Single Molecules by Dielectrophoresis, Physical Review Letters 95 (2005) 128102.

[97] J.D. Beck, L. Shang, B. Li, M.S. Marcus, R.J. Hamers, Discrimination between Bacillus species by impedance analysis of individual dielectrophoretically positioned spores, Analytical Chemistry 80 (2008) 3757-3761.

[98] Q. Ramadan, V. Samper, D. Poenar, Z. Liang, C. Yu, T.M. Lim, Simultaneous cell lysis and bead trapping in a continuous flow microfluidic device, Sensors and Actuators B-Chemical 113 (2006) 944-955.

[99] S. Agarwal, A. Sebastian, L.M. Forrester, G.H. Markx, Formation of embryoid bodies using dielectrophoresis, Biomicrofluidics 6 (2012).

[100] N.G. Green, H. Morgan, Separation of submicrometre particles using a combination of dielectrophoretic and electrohydrodynamic forces, Journal of Physics D-Applied Physics 31 (1998) L25-L30.

[101] R. Pethig, Y. Huang, X.B. Wang, J.P.H. Burt, POSITIVE AND NEGATIVE DIELECTROPHORETIC COLLECTION OF COLLOIDAL PARTICLES USING INTERDIGITATED CASTELLATED MICROELECTRODES, Journal of Physics D-Applied Physics 25 (1992) 881-888.

[102] A.C. Sabuncu, J.A. Liu, S.J. Beebe, A. Beskok, Dielectrophoretic separation of mouse melanoma clones, Biomicrofluidics 4 (2010).

[103] P.R.C. Gascoyne, X.B. Wang, Y. Huang, F.F. Becker, Dielectrophoretic separation of cancer cells from blood, Ieee Transactions on Industry Applications 33 (1997) 670-678.

[104] F.F. Becker, X.B. Wang, Y. Huang, R. Pethig, J. Vykoukal, P.R.C. Gascoyne, SEPARATION OF HUMAN BREAST-CANCER CELLS FROM BLOOD BY DIFFERENTIAL DIELECTRIC 
AFFINITY, Proceedings of the National Academy of Sciences of the United States of America 92 (1995) 860-864.

[105] K. Zhu, A.S. Kaprelyants, E.G. Salina, G.H. Markx, Separation by dielectrophoresis of dormant and nondormant bacterial cells of Mycobacterium smegmatis, Biomicrofluidics 4 (2010).

[106] W. Choi, J.-S. Kim, D.-H. Lee, K.-K. Lee, D.-B. Koo, J.-K. Park, Dielectrophoretic oocyte selection chip for in vitro fertilization, Biomedical Microdevices 10 (2008) 337-345.

[107] C.-H. Tai, S.-K. Hsiung, C.-Y. Chen, M.-L. Tsai, G.-B. Lee, Automatic microfluidic platform for cell separation and nucleus collection, Biomedical Microdevices 9 (2007) 533-543.

[108] S. Rajaraman, H. Noh, P.J. Hesketh, D.S. Gottfried, Rapid, low cost microfabrication technologies toward realization of devices for dielectrophoretic manipulation of particles and nanowires, Sensors and Actuators B-Chemical 114 (2006) 392-401.

[109] C. Zhang, K. Khoshmanesh, F.J. Tovar-Lopez, A. Mitchell, W. Wlodarski, K. Klantar-zadeh, Dielectrophoretic separation of carbon nanotubes and polystyrene microparticles, Microfluidics and Nanofluidics 7 (2009) 633-645.

[110] T. Yasukawa, M. Suzuki, H. Shiku, T. Matsue, Control of the microparticle position in the channel based on dielectrophoresis, Sensors and Actuators B-Chemical 142 (2009) 400-403.

[111] J. Suehiro, Fabrication and characterization of nanomaterial-based sensors using dielectrophoresis, Biomicrofluidics 4 (2010).

[112] H.B. Li, R. Bashir, Dielectrophoretic separation and manipulation of live and heat-treated cells of Listeria on microfabricated devices with interdigitated electrodes, Sensors and Actuators B-Chemical 86 (2002) 215-221.

[113] Y. Li, C. Dalton, H.J. Crabtree, G. Nilsson, K.V.I.S. Kaler, Continuous dielectrophoretic cell separation microfluidic device, Lab on a Chip 7 (2007) 239-248.

[114] J. Auerswald, H.F. Knapp, Quantitative assessment of dielectrophoresis as a micro fluidic retention and separation technique for beads and human blood erythrocytes, Microelectronic Engineering 67-8 (2003) 879-886.

[115] C.L. Asbury, A.H. Diercks, G. van den Engh, Trapping of DNA by dielectrophoresis, Electrophoresis 23 (2002) 2658-2666.

[116] N. Gadish, J. Voldman, High-throughput positive-dielectrophoretic bioparticle microconcentrator, Analytical Chemistry 78 (2006) 7870-7876.

[117] G. Giraud, R. Pethig, H. Schulze, G. Henihan, J.G. Terry, A. Menachery, I. Ciani, D. Corrigan, C.J. Campbell, A.R. Mount, P. Ghazal, A.J. Walton, J. Crain, T.T. Bachmann, Dielectrophoretic manipulation of ribosomal RNA, Biomicrofluidics 5 (2011).

[118] L. Wu, L. Lanry Yung, K. Lim, Dielectrophoretic capture voltage spectrum for measurement of dielectric properties and separation of cancer cells, Biomicrofluidics 6 (2012).

[119] H. Narayanan Unni, D. Hartono, L. Yue Lanry Yung, M. Mah-Lee Ng, H. Pueh Lee, B. Cheong Khoo, K.M. Lim, Characterization and separation of Cryptosporidium and Giardia cells using on-chip dielectrophoresis, Biomicrofluidics 6 (2012).

[120] M. Suzuki, T. Yasukawa, H. Shiku, T. Matsue, Negative dielectrophoretic patterning with colloidal particles and encapsulation into a hydrogel, Langmuir 23 (2007) 4088-4094.

[121] S. Park, A. Beskok, Alternating current electrokinetic motion of colloidal particles on interdigitated microelectrodes, Analytical Chemistry 80 (2008) 2832-2841.

[122] N. Crews, J. Darabi, P. Voglewede, F. Guo, A. Bayoumi, An analysis of interdigitated electrode geometry for dielectrophoretic particle transport in micro-fluidics, Sensors and Actuators B-Chemical 125 (2007) 672-679.

[123] J. Suehiro, M. Shutou, T. Hatano, M. Hara, High sensitive detection of biological cells using dielectrophoretic impedance measurement method combined with electropermeabilization, Sensors and Actuators B-Chemical 96 (2003) 144-151.

[124] J. Suehiro, R. Yatsunami, R. Hamada, M. Hara, Quantitative estimation of biological cell concentration suspended in aqueous medium by using dielectrophoretic impedance measurement method, Journal of Physics D-Applied Physics 32 (1999) 2814-2820.

[125] J. Vykoukal, D.M. Vykoukal, S. Freyberg, E.U. Alt, P.R.C. Gascoyne, Enrichment of putative stem cells from adipose tissue using dielectrophoretic field-flow fractionation, Lab on a Chip 8 (2008) 1386-1393.

[126] S. Shim, K. Stemke-Hale, J. Noshari, F.F. Becker, P.R.C. Gascoyne, Dielectrophoresis has broad applicability to marker-free isolation of tumor cells from blood by microfluidic systems, Biomicrofluidics 7 (2013).

[127] S. Shim, K. Stemke-Hale, A.M. Tsimberidou, J. Noshari, T.E. Anderson, P.R.C. Gascoyne, Antibodyindependent isolation of circulating tumor cells by continuous-flow dielectrophoresis, Biomicrofluidics 7 (2013). 
[128] E.T. Lagally, S.H. Lee, H.T. Soh, Integrated microsystem for dielectrophoretic cell concentration and genetic detection, Lab on a Chip 5 (2005) 1053-1058.

[129] U. Kim, J. Qian, S.A. Kenrick, P.S. Daugherty, H.T. Soh, Multitarget Dielectrophoresis Activated Cell Sorter, Analytical Chemistry 80 (2008) 8656-8661.

[130] J.G. Kralj, M.T.W. Lis, M.A. Schmidt, K.F. Jensen, Continuous dielectrophoretic size-based particle sorting, Analytical Chemistry 78 (2006) 5019-5025.

[131] M.D. Vahey, J. Voldman, An equilibrium method for continuous-flow cell sorting using dielectrophoresis, Analytical Chemistry 80 (2008) 3135-3143.

[132] R.S. Kuczenski, H.C. Chang, A. Revzin, Dielectrophoretic microfluidic device for the continuous sorting of Escherichia coli from blood cells, Biomicrofluidics 5 (2011).

[133] M.S. Pommer, Y. Zhang, N. Keerthi, D. Chen, J.A. Thomson, C.D. Meinhart, H.T. Soh, Dielectrophoretic separation of platelets from diluted whole blood in microfluidic channels, Electrophoresis 29 (2008) 1213-1218.

[134] K.-H. Han, A.B. Frazier, Lateral-driven continuous dielectrophoretic microseparators for blood cells suspended in a highly conductive medium, Lab on a Chip 8 (2008) 1079-1086.

[135] K. Khoshmanesh, C. Zhang, S. Nahavandi, F.J. Tovar-Lopez, S. Baratchi, A. Mitchell, K. Kalantar-Zadeh, Size based separation of microparticles using a dielectrophoretic activated system, Journal of Applied Physics 108 (2010)

[136] K.-H. Han, S.-I. Han, A.B. Frazier, Lateral displacement as a function of particle size using a piecewise curved planar interdigitated electrode array, Lab on a Chip 9 (2009) 2958-2964.

[137] K. Khoshmanesh, C. Zhang, F.J. Tovar-Lopez, S. Nahavandi, S. Baratchi, K. Kalantar-zadeh, A. Mitchell, Dielectrophoretic manipulation and separation of microparticles using curved microelectrodes, Electrophoresis 30 (2009) 3707-3717.

[138] K. Khoshmanesh, C. Zhang, F.J. Tovar-Lopez, S. Nahavandi, S. Baratchi, A. Mitchell, K. Kalantar-Zadeh, Dielectrophoretic-activated cell sorter based on curved microelectrodes, Microfluidics and Nanofluidics 9 (2010) 411-426.

[139] K. Khoshmanesh, C. Zhang, S. Nahavandi, F.J. Tovar-Lopez, S. Baratchi, Z. Hu, A. Mitchell, K. Kalantar-zadeh, Particle trapping using dielectrophoretically patterned carbon nanotubes, Electrophoresis 31 (2010) 1366-1375.

[140] K. Khoshmanesh, C. Zhang, S. Nahavandi, S. Baratchi, A. Mitchell, K. Kalantar-zadeh, Dielectrophoretically patterned carbon nanotubes to sort microparticles, Electrophoresis 31 (2010) 3380-3390.

[141] C.P. Jen, H.H. Chang, A handheld preconcentrator for the rapid collection of cancerous cells using dielectrophoresis generated by circular microelectrodes in stepping electric fields, Biomicrofluidics 5 (2011).

[142] A. Rosenthal, J. Voldman, Dielectrophoretic traps for single-particle patterning, Biophysical Journal 88 (2005) 2193-2205.

[143] N. Mittal, A. Rosenthal, J. Voldman, NDEP microwells for single-cell patterning in physiological media, Lab on a Chip 7 (2007) 1146-1153.

[144] M. Koklu, S. Park, S.D. Pillai, A. Beskok, Negative dielectrophoretic capture of bacterial spores in food matrices, Biomicrofluidics 4 (2010).

[145] L.-S. Jang, P.-H. Huang, K.-C. Lan, Single-cell trapping utilizing negative dielectrophoretic quadrupole and microwell electrodes, Biosensors \& Bioelectronics 24 (2009) 3637-3644.

[146] R. Krishnan, B.D. Sullivan, R.L. Mifflin, S.C. Esener, M.J. Heller, Alternating current electrokinetic separation and detection of DNA nanoparticles in high-conductance solutions, Electrophoresis 29 (2008) 1765-1774.

[147] N. Manaresi, A. Romani, G. Medoro, L. Altomare, A. Leonardi, M. Tartagni, R. Guerrieri, A CMOS chip for individual cell manipulation and detection, Ieee Journal of Solid-State Circuits 38 (2003) 22972305.

[148] T.P. Hunt, D. Issadore, R.M. Westervelt, Integrated circuit/microfluidic chip to programmably trap and move cells and droplets with dielectrophoresis, Lab on a Chip 8 (2008) 81-87.

[149] P.R.C. Gascoyne, J.V. Vykoukal, J.A. Schwartz, T.J. Anderson, D.M. Vykoukal, K.W. Current, C. McConaghy, F.F. Becker, C. Andrews, Dielectrophoresis-based programmable fluidic processors, Lab on a Chip 4 (2004) 299-309.

[150] A.B. Fuchs, A. Romani, D. Freida, G. Medoro, M. Abonnenc, L. Altomare, I. Chartier, D. Guergour, C. Villiers, P.N. Marche, M. Tartagni, R. Guerrieri, F. Chatelain, N. Manaresi, Electronic sorting and recovery of single live cells from microlitre sized samples, Lab on a Chip 6 (2006) 121-126.

[151] R.S. Thomas, H. Morgan, N.G. Green, Negative DEP traps for single cell immobilisation, Lab on a Chip 9 (2009) 1534-1540. 
[152] R.S.W. Thomas, P.D. Mitchell, R.O.C. Oreffo, H. Morgan, Trapping single human osteoblast-like cells from a heterogeneous population using a dielectrophoretic microfluidic device, Biomicrofluidics 4 (2010).

[153] K. Zhu, A.S. Kaprelyants, E.G. Salina, M. Schuler, G.H. Markx, Construction by dielectrophoresis of microbial aggregates for the study of bacterial cell dormancy, Biomicrofluidics 4 (2010).

[154] K. Ino, H. Shiku, F. Ozawa, T. Yasukawa, T. Matsue, Manipulation of Microparticles for Construction of Array Patterns by Negative Dielectrophoresis Using Multilayered Array and Grid Electrodes, Biotechnology and Bioengineering 104 (2009) 709-718.

[155] B.M. Taff, J. Voldman, A scalable addressable positive-dielectrophoretic cell-sorting array, Analytical Chemistry 77 (2005) 7976-7983.

[156] E.M. Melvin, B.R. Moore, K.H. Gilchrist, S. Grego, O.D. Velev, On-chip collection of particles and cells by $\mathrm{AC}$ electroosmotic pumping and dielectrophoresis using asymmetric microelectrodes, Biomicrofluidics 5 (2011).

[157] C. Suscillon, O.D. Velev, V.I. Slaveykova, Alternating current-dielectrophoresis driven on-chip collection and chaining of green microalgae in freshwaters, Biomicrofluidics 7 (2013).

[158] M. Nikolic-Jaric, T. Cabel, E. Salimi, A. Bhide, K. Braasch, M. Butler, G.E. Bridges, D.J. Thomson, Differential electronic detector to monitor apoptosis using dielectrophoresis-induced translation of flowing cells (dielectrophoresis cytometry), Biomicrofluidics 7 (2013).

[159] T. Yasukawa, M. Suzuki, T. Sekiya, H. Shiku, T. Matsue, Flow sandwich-type immunoassay in microfluidic devices based on negative dielectrophoresis, Biosensors \& Bioelectronics 22 (2007) 27302736.

[160] M. Li, S. Li, W. Cao, W. Li, W. Wen, G. Alici, Improved concentration and separation of particles in a 3D dielectrophoretic chip integrating focusing, aligning and trapping, Microfluidics and Nanofluidics 14 (2013) 527-539.

[161] S. Li, M. Li, K. Bougot-Robin, W. Cao, I.Y.Y. Chau, W. Li, W. Wen, High-throughput particle manipulation by hydrodynamic, electrokinetic and dielectrophoretic effects in an integrated microfluidic chip, Biomicrofluidics (2013).

[162] J. Park, B. Kim, S.K. Choi, S. Hong, S.H. Lee, K.I. Lee, An efficient cell separation system using 3Dasymmetric microelectrodes, Lab on a Chip 5 (2005) 1264-1270.

[163] F. Grom, J. Kentsch, T. Muller, T. Schnelle, M. Stelzle, Accumulation and trapping of hepatitis A virus particles by electrohydrodynamic flow and dielectrophoresis, Electrophoresis 27 (2006) 1386-1393.

[164] M.S. Jaeger, K. Uhlig, T. Schnelle, T. Mueller, Contact-free single-cell cultivation by negative dielectrophoresis, Journal of Physics D-Applied Physics 41 (2008).

[165] C.H. Yu, J. Vykoukal, D.M. Vykoukal, J.A. Schwartz, L. Shi, P.R.C. Gascoyne, A three-dimensional dielectrophoretic particle focusing channel for microcytometry applications, Journal of Microelectromechanical Systems 14 (2005) 480-487.

[166] J. Voldman, M.L. Gray, M. Toner, M.A. Schmidt, A microfabrication-based dynamic array cytometer, Analytical Chemistry 74 (2002) 3984-3990.

[167] C. Iliescu, L. Yu, F.E.H. Tay, B. Chen, Bidirectional field-flow particle separation method in a dielectrophoretic chip with 3D electrodes, Sensors and Actuators B-Chemical 129 (2008) 491-496.

[168] T.P. Hunt, H. Lee, R.M. Westervelt, Addressable micropost array for the dielectrophoretic manipulation of particles in fluid, Applied Physics Letters 85 (2004) 6421-6423.

[169] J. Voldman, M. Toner, M.L. Gray, M.A. Schmidt, Design and analysis of extruded quadrupolar dielectrophoretic traps, Journal of Electrostatics 57 (2003) 69-90.

[170] B.Y. Park, M.J. Madou, 3-D electrode designs for flow-through dielectrophoretic systems, Electrophoresis 26 (2005) 3745-3757.

[171] M. del Carmen Jaramillo, E. Torrents, R. Martinez-Duarte, M.J. Madou, A. Juarez, On-line separation of bacterial cells by carbon-electrode dielectrophoresis, Electrophoresis 31 (2010) 2921-2928.

[172] R. Martinez-Duarte, R.A. Gorkin, III, K. Abi-Samra, M.J. Madou, The integration of 3D carbon-electrode dielectrophoresis on a CD-like centrifugal microfluidic platform, Lab on a Chip 10 (2010) 1030-1043.

[173] F.E.H. Tay, L. Yu, A.J. Pang, C. Iliescu, Electrical and thermal characterization of a dielectrophoretic chip with 3D electrodes for cells manipulation, Electrochimica Acta 52 (2007) 2862-2868.

[174] L. Yu, C. Iliescu, G. Xu, F.E.H. Tay, Sequential field-flow cell separation method in a dielectrophoretic chip with 3-D electrodes, Journal of Microelectromechanical Systems 16 (2007) 1120-1129.

[175] L. Wang, J. Lu, S.A. Marukenko, E.S. Monuki, L.A. Flanagan, A.P. Lee, Dual frequency dielectrophoresis with interdigitated sidewall electrodes for microfluidic flow-through separation of beads and cells, Electrophoresis 30 (2009) 782-791.

[176] B. Cetin, D. Li, Lab-on-a-chip device for continuous particle and cell separation based on electrical properties via alternating current dielectrophoresis, Electrophoresis 31 (2010) 3035-3043. 
[177] N. Piacentini, G. Mernier, R. Tornay, P. Renaud, Separation of platelets from other blood cells in continuous-flow by dielectrophoresis field-flow-fractionation, Biomicrofluidics 5 (2011).

[178] L. Wang, L.A. Flanagan, N.L. Jeon, E. Monuki, A.P. Lee, Dielectrophoresis switching with vertical sidewall electrodes for microfluidic flow cytometry, Lab on a Chip 7 (2007) 1114-1120.

[179] N. Lewpiriyawong, C. Yang, AC-dielectrophoretic characterization and separation of submicron and micron particles using sidewall AgPDMS electrodes, Biomicrofluidics 6 (2012).

[180] N. Lewpiriyawong, K. Kandaswamy, C. Yang, V. Ivanov, R. Stocker, Microfluidic Characterization and Continuous Separation of Cells and Particles Using Conducting Poly(dimethyl siloxane) Electrode Induced Alternating Current-Dielectrophoresis, Analytical Chemistry 83 (2011) 9579-9585.

[181] B. Cetin, Y. Kang, Z. Wu, D. Li, Continuous particle separation by size via AC-dielectrophoresis using a lab-on-a-chip device with 3-D electrodes, Electrophoresis 30 (2009) 766-772.

[182] N. Hu, J. Yang, S. Qian, S.W. Joo, X. Zheng, A cell electrofusion microfluidic device integrated with 3D thin-film microelectrode arrays, Biomicrofluidics 5 (2011).

[183] T. Braschler, N. Demierre, E. Nascimento, T. Silva, A.G. Oliva, P. Renaud, Continuous separation of cells by balanced dielectrophoretic forces at multiple frequencies, Lab on a Chip 8 (2008) 280-286.

[184] A. Valero, T. Braschler, N. Demierre, P. Renaud, A miniaturized continuous dielectrophoretic cell sorter and its applications, Biomicrofluidics 4 (2010).

[185] E.M. Nascimento, N. Nogueira, T. Silva, T. Braschler, N. Demierre, P. Renaud, A.G. Oliva, Dielectrophoretic sorting on a microfabricated flow cytometer: Label free separation of Babesia bovis infected erythrocytes, Bioelectrochemistry 73 (2008) 123-128.

[186] R.L. Tornay, T. Braschler, N. Demierre, B. Steitz, A. Finka, H. Hofmann, J.A. Hubbell, P. Renaud, Dielectrophoresis-based particle exchanger for the manipulation and surface functionalization of particles, Lab on a Chip 8 (2008) 267-273.

[187] B.H. Lapizco-Encinas, B.A. Simmons, E.B. Cummings, Y. Fintschenko, Dielectrophoretic concentration and separation of live and dead bacteria in an array of insulators, Analytical Chemistry 76 (2004) 15711579.

[188] C.F. Chou, J.O. Tegenfeldt, O. Bakajin, S.S. Chan, E.C. Cox, N. Darnton, T. Duke, R.H. Austin, Electrodeless dielectrophoresis of single- and double-stranded DNA, Biophysical Journal 83 (2002) 2170-2179.

[189] C.F. Chou, F. Zenhausern, Electrodeless dielectrophoresis for micro total analysis systems, Ieee Engineering in Medicine and Biology Magazine 22 (2003) 62-67.

[190] R.C. Gallo-Villanueva, C.E. Rodriguez-Lopez, R.I. Diaz-de-la-Garza, C. Reyes-Betanzo, B.H. LapizcoEncinas, DNA manipulation by means of insulator-based dielectrophoresis employing direct current electric fields, Electrophoresis 30 (2009) 4195-4205.

[191] B.H. Lapizco-Encinas, S. Ozuna-Chacon, M. Rito-Palomares, Protein manipulation with insulator-based dielectrophoresis and direct current electric fields, Journal of Chromatography A 1206 (2008) 45-51.

[192] A. Nakano, F. Camacho-Alanis, T.C. Chao, A. Ros, Tuning direct current streaming dielectrophoresis of proteins, Biomicrofluidics 6 (2012).

[193] B.H. Lapizco-Encinas, R.V. Davalos, B.A. Simmons, E.B. Cummings, Y. Fintschenko, An insulatorbased (electrodeless) dielectrophoretic concentrator for microbes in water, Journal of Microbiological Methods 62 (2005) 317-326.

[194] B.H. Lapizco-Encinas, B.A. Simmons, E.B. Cummings, Y. Fintschenko, Insulator-based dielectrophoresis for the selective concentration and separation of live bacteria in water, Electrophoresis 25 (2004) 1695 1704.

[195] E.B. Cummings, A.K. Singh, Dielectrophoresis in microchips containing arrays of insulating posts: Theoretical and experimental results, Analytical Chemistry 75 (2003) 4724-4731.

[196] S. Ozuna-Chacon, B.H. Lapizco-Encinas, M. Rito-Palomares, S.O. Martinez-Chapa, C. Reyes-Betanzo, Performance characterization of an insulator-based dielectrophoretic microdevice, Electrophoresis 29 (2008) 3115-3122.

[197] C. Iliescu, G.L. Xu, P.L. Ong, K.J. Leck, Dielectrophoretic separation of biological samples in a 3D filtering chip, Journal of Micromechanics and Microengineering 17 (2007) S128-S136.

[198] C. Iliescu, G. Xu, F.C. Loe, P.L. Ong, F.E.H. Tay, A 3-D dielectrophoretic filter chip, Electrophoresis 28 (2007) 1107-1114.

[199] K.H. Kang, Y.J. Kang, X.C. Xuan, D.Q. Li, Continuous separation of microparticies by size with direct current-dielectrophoresis, Electrophoresis 27 (2006) 694-702.

[200] L.M. Barrett, A.J. Skulan, A.K. Singh, E.B. Cummings, G.J. Fiechtner, Dielectrophoretic manipulation of particles and cells using insulating ridges in faceted prism microchannels, Analytical Chemistry 77 (2005) 6798-6804.

[201] K.H. Kang, X.C. Xuan, Y.J. Kang, D.Q. Li, Effects of dc-dielectrophoretic force on particle trajectories in microchannels, Journal of Applied Physics 99 (2006). 
[202] Y.J. Kang, D.Q. Li, S.A. Kalams, J.E. Eid, DC-Dielectrophoretic separation of biological cells by size, Biomedical Microdevices 10 (2008) 243-249.

[203] G.O.F. Parikesit, A.P. Markesteijn, O.M. Piciu, A. Bossche, J. Westerweel, I.T. Young, Y. Garini, Sizedependent trajectories of DNA macromolecules due to insulative dielectrophoresis in submicrometerdeep fluidic channels, Biomicrofluidics 2 (2008).

[204] C. Church, J. Zhu, G. Huang, T.R. Tzeng, X. Xuan, Integrated electrical concentration and lysis of cells in a microfluidic chip, Biomicrofluidics 4 (2010).

[205] N. Lewpiriyawong, C. Yang, Y.C. Lam, Dielectrophoretic manipulation of particles in a modified microfluidic $\mathrm{H}$ filter with multi-insulating blocks, Biomicrofluidics 2 (2008).

[206] I. Barbulovic-Nad, X.C. Xuan, J.S.H. Lee, D.Q. Li, DC-dielectrophoretic separation of microparticles using an oil droplet obstacle, Lab on a Chip 6 (2006) 274-279.

[207] B.G. Hawkins, A.E. Smith, Y.A. Syed, B.J. Kirby, Continuous-flow particle separation by 3D insulative dielectrophoresis using coherently shaped, dc-biased, ac electric fields, Analytical Chemistry 79 (2007) 7291-7300.

[208] C.-P. Jen, C.-H. Weng, C.-T. Huang, Three-dimensional focusing of particles using negative dielectrophoretic force in a microfluidic chip with insulating microstructures and dual planar microelectrodes, Electrophoresis 32 (2011) 2428-2435.

[209] C.-P. Jen, C.-T. Huang, C.-H. Weng, Focusing of biological cells utilizing negative dielectrophoretic force generated by insulating structures, Microelectronic Engineering 87 (2010) 773-777.

[210] C.-P. Jen, C.-T. Huang, H.-Y. Shih, Hydrodynamic separation of cells utilizing insulator-based dielectrophoresis, Microsystem Technologies-Micro-and Nanosystems-Information Storage and Processing Systems 16 (2010) 1097-1104.

[211] C. Huang, C. Weng, C. Jen, Three-dimensional cellular focusing utilizing a combination of insulatorbased and metallic dielectrophoresis, Biomicrofluidics 5 (2011).

[212] S. Patel, D. Showers, P. Vedantam, T.R. Tzeng, S. Qian, X. Xuan, Microfluidic separation of live and dead yeast cells using reservoir-based dielectrophoresis, Biomicrofluidics 6 (2012).

[213] M.D. Pysher, M.A. Hayes, Electrophoretic and dielectrophoretic field gradient technique for separating bioparticles, Analytical Chemistry 79 (2007) 4552-4557.

[214] P.V. Jones, S.J.R. Staton, M.A. Hayes, Blood cell capture in a sawtooth dielectrophoretic microchannel, Analytical and Bioanalytical Chemistry 401 (2011) 2103-2111.

[215] K.P. Chen, J.R. Pacheco, M.A. Hayes, S.J.R. Staton, Insulator-based dielectrophoretic separation of small particles in a sawtooth channel, Electrophoresis 30 (2009) 1441-1448.

[216] C. Church, J. Zhu, J. Nieto, G. Keten, E. Ibarra, X. Xuan, Continuous particle separation in a serpentine microchannel via negative and positive dielectrophoretic focusing, Journal of Micromechanics and Microengineering 20 (2010).

[217] M. Li, S. Li, W. Cao, W. Li, W. Wen, G. Alici, Continuous particle focusing in a waved microchannel using negative dc dielectrophoresis, Journal of Micromechanics and Microengineering 22 (2012).

[218] J. Zhu, T.-R.J. Tzeng, X. Xuan, Continuous dielectrophoretic separation of particles in a spiral microchannel, Electrophoresis 31 (2010) 1382-1388.

[219] J. Zhu, T.-R.J. Tzeng, G. Hu, X. Xuan, DC dielectrophoretic focusing of particles in a serpentine microchannel, Microfluidics and Nanofluidics 7 (2009) 751-756.

[220] C. Church, J. Zhu, G. Wang, T.-R.J. Tzeng, X. Xuan, Electrokinetic focusing and filtration of cells in a serpentine microchannel, Biomicrofluidics 3 (2009).

[221] J. Zhu, R.C. Canter, G. Keten, P. Vedantam, T.-R.J. Tzeng, X. Xuan, Continuous-flow particle and cell separations in a serpentine microchannel via curvature-induced dielectrophoresis, Microfluidics and Nanofluidics 11 (2011) 743-752.

[222] C. Church, J. Zhu, X. Xuan, Negative dielectrophoresis-based particle separation by size in a serpentine microchannel, Electrophoresis 32 (2011) 527-531.

[223] J. Zhu, X. Xuan, Particle electrophoresis and dielectrophoresis in curved microchannels, Journal of Colloid and Interface Science 340 (2009) 285-290.

[224] J.J. Zhu, X.C. Xuan, Curvature-induced dielectrophoresis for continuous separation of particles by charge in spiral microchannels, Biomicrofluidics 5 (2011).

[225] L. Zhang, F. Tatar, P. Turmezei, J. Bastemeijer, J.R. Mollinger, O. Piciu, A. Bossche, Continuous Electrodeless Dielectrophoretic Separation in a Circular Channel, International Mems Conference 2006, 2006, pp. 527-532.

[226] M. Li, S. Li, W. Li, W. Wen, G. Alici, Continuous manipulation and separation of particles using combined obstacle-and curvature-induced DC dielectrophoresis, Electrophoresis (2013).

[227] Y.-K. Cho, S. Kim, K. Lee, C. Park, J.-G. Lee, C. Ko, Bacteria concentration using a membrane type insulator-based dielectrophoresis in a plastic chip, Electrophoresis 30 (2009) 3153-3159. 
[228] H. Shafiee, M.B. Sano, E.A. Henslee, J.L. Caldwell, R.V. Davalos, Selective isolation of live/dead cells using contactless dielectrophoresis (cDEP), Lab on a Chip 10 (2010) 438-445.

[229] E.A. Henslee, M.B. Sano, A.D. Rojas, E.M. Schmelz, R.V. Davalos, Selective concentration of human cancer cells using contactless dielectrophoresis, Electrophoresis 32 (2011) 2523-2529.

[230] M.B. Sano, J.L. Caldwell, R.V. Davalos, Modeling and development of a low frequency contactless dielectrophoresis (cDEP) platform to sort cancer cells from dilute whole blood samples, Biosensors \& Bioelectronics 30 (2011) 13-20.

[231] A. Salmanzadeh, L. Romero, H. Shafiee, R.C. Gallo-Villanueva, M.A. Stremler, S.D. Cramer, R.V. Davalos, Isolation of prostate tumor initiating cells (TICs) through their dielectrophoretic signature, Lab on a Chip 12 (2012) 182-189.

[232] A. Salmanzadeh, H. Kittur, M.B. Sano, P. C Roberts, E.M. Schmelz, R.V. Davalos, Dielectrophoretic differentiation of mouse ovarian surface epithelial cells, macrophages, and fibroblasts using contactless dielectrophoresis, Biomicrofluidics 6 (2012) 24104-2410413.

[233] A. Salmanzadeh, M.B. Sano, R.C. Gallo-Villanueva, P.C. Roberts, E.M. Schmelz, R.V. Davalos, Investigating dielectric properties of different stages of syngeneic murine ovarian cancer cells, Biomicrofluidics 7 (2013). 